JOURNAL OF THE

AMERICAN MATHEMATICAL SOCIETY

Volume 11, Number 3, July 1998, Pages 601-634

S 0894-0347(98)00262-8

\title{
ON THE AFFINE HEAT EQUATION FOR NON-CONVEX CURVES
}

\author{
SIGURD ANGENENT, GUILLERMO SAPIRO, AND ALLEN TANNENBAUM
}

\section{INTRODUCTION}

In the past several years, there has been much research devoted to the study of evolutions of plane curves where the velocity of the evolving curve is given by the Euclidean curvature vector. This evolution appears in a number of different pure and applied areas such as differential geometry, crystal growth, and computer vision. See for example $[4,5,6,15,16,17,19,20,35]$ and the references therein.

As is well known, this Euclidean curvature evolution is a "Euclidean curve shortening" process, precisely because the flow lines in the space of curves are tangent to the gradient of the length functional. Therefore, the curve perimeter is shrinking as fast as possible [17]. The behavior of an embedded curve evolving according to this flow has been well-studied. Gage and Hamilton prove that a convex embedded smooth initial curve converges to a round point under this evolution [13, 14, 15]. Grayson [16] has shown that a non-convex embedded curve converges to a convex one, and from there to a round point according to the Gage and Hamilton result. Since this evolution is based on Euclidean invariant concepts (Euclidean curvature vector), the solution is invariant only under rigid plane motions (i.e., the group of proper Euclidean motions in $\mathbf{R}^{2}$ generated by rotations and translations). This equation has also been called the geometric heat equation.

Recently, the affine analogue of the Euclidean curve shortening flow was considered for convex curves [30]. In this case, the velocity of the evolving curve is given by the affine normal vector. The investigation of this type of evolution was motivated by the search for affine invariant flows in computer vision and image processing $[32,31]$. Among the results proven in this work is that when a curve is evolving according to this flow, the area shrinks as fast as possible with respect to a certain affine metric [27]. Since the affine distance is based on area [8], in this sense, this evolution is an affine shortening flow. (See our discussion in Section 3 as well.) We have also shown that any convex plane curve converges to an elliptical point (defined relative to the corresponding family of normalized dilated curves) when the deformation is given by this affine shortening. These results make this evolution the affine analogue of the Euclidean curve shortening for convex curves.

Received by the editors April 24, 1997 and, in revised form, January 20, 1998.

1991 Mathematics Subject Classification. Primary 35K22, 53A15, 58G11.

This work was supported in part by grants from the National Science Foundation DMS9058492, ECS-9122106, ECS-99700588, NSF-LIS, by the Air Force Office of Scientific Research AF/F49620-94-1-00S8DEF, AF/F49620-94-1-0461, AF/F49620-98-1-0168, by the Army Research Office DAAL03-92-G-0115, DAAH04-94-G-0054, DAAH04-93-G-0332, MURI Grant, Office of Naval Research ONR-N00014-97-1-0509, and by the Rothschild Foundation-Yad Hanadiv. 
We will also refer to this flow as the affine invariant geometric equation; see our discussion in Section 3 below. Independently, Alvarez et al. [1, 2] have considered an equivalent model from the point of view of viscosity solutions. We should also note that very recently, Andrews in [3] has generalized the results of [30] to convex hypersurfaces moving according to their affine normal.

The goal of this work is to extend the affine shortening flow to non-convex curves. One of the key problems to be overcome is the fact that the basic invariants of affine differential geometry are not defined for non-convex curves. In particular, we have to define the evolution velocity vector for inflection points. This extension must keep the affine invariance property, and must reduce to the original affine flow for convex curves. In this note, a natural extension is presented for which these conditions hold. The key step in this extension is the fact that the Euclidean normal component of the affine normal is given by $\kappa^{1 / 3} \mathcal{N}$ where $\kappa$ denotes the curvature and $\mathcal{N}$ the (ordinary) Euclidean unit normal [30], and in fact it turns out that the evolution is defined by taking the normal velocity vector to be precisely $\kappa^{1 / 3} \mathcal{N}$. Using techniques based on $[4,5,16,30,32]$, we show that any simple closed curve will shrink to a point under Affine Curve Shortening. Moreover, we prove that the total curvature of any such solution tends to $2 \pi$. See Theorem 15.1.

We now summarize the contents of this paper. In Section 2, we discuss some of the basic facts from affine differential geometry that we will need in the sequel. In Section 3, we introduce the affine invariant heat equation for convex curves and sketch the relevant background from [30]. Section 4 is concerned with the extension of this flow to non-convex curves. In Section 5, we prove short-term existence using a version of Nash-Moser iteration. In Section 6, we prove uniqueness for $C^{2}$ initial data, and then in Section 7 establish a version of the weak and strong maximum principles for Affine Curve Shortening. Section 8 contains bounds on the curvature which we will need for convergence. In Section 9, we give a bound on the number of maximal convex and concave arcs.

Counting intersections of solutions is a fundamental tool in the study of onedimensional diffusion equations. In Section 10, we recall the weak version of the intersection comparison theorem due to Matano [21] and note that it applies to the affine heat flow. We also show that the strong maximum principle is not valid for the affine heat equation, and only a weak maximum principle holds. In Section 11, we develop the technique of evolving foliated rectangles which allows us to rule out the formation of certain singularities in Sections 12 and 13. Then in Section 14, we give an affine version of the Grayson $\delta$-whisker lemma, which we use in our proof of the main result on the convergence of a non-convex initial curve to an elliptical point in Section 15.

\section{Sketch of AfFine Differential GeOMEtry}

In this section, we summarize some of the basic notions from affine differential geometry that we will need in the sequel. Our treatment is based on the classical works $[8,18]$.

A general affine transformation in the plane $\left(\mathbf{R}^{2}\right)$ is defined as

$$
\tilde{X}=A X+B
$$

where $X \in \mathbf{R}^{2}$ is a vector, $A \in \mathrm{GL}_{2}^{+}(\mathbf{R})$ (the group of invertible real $2 \times 2$ matrices with positive determinant) is the affine matrix, and $B \in \mathbf{R}^{2}$ is a translation vector. 
It is easy to show that transformations of the type (1) form a real algebraic group $\mathcal{A}$, called the group of proper affine motions or full affine group. We will also consider the case of when we restrict $A \in \mathrm{SL}_{2}(\mathbf{R})$ (i.e., the determinant of $A$ is 1 ), in which case (1) gives us the group of special affine motions or special affine group, $\mathcal{A}_{s p}$.

We now very briefly recall the notion of "invariant" $[10,18]$. A quantity $Q$ is called a relative invariant of a Lie group $\mathcal{G}$ if whenever $Q$ transforms into $\tilde{Q}$ by any transformation $g \in \mathcal{G}$, we obtain $\tilde{Q}=\Psi Q$, where $\Psi$ is a function of $g$ alone. If $\Psi=1$ for all $g \in \mathcal{G}, Q$ is called an invariant [10]. Differential invariants may be defined in terms of the prolongation of the action of the relevant transformation group to the appropriate jet space $[22,23]$. Affine differential geometry is concerned with the differential invariants of the special affine group $\mathcal{A}_{s p}$. Thus all the affine invariants we will discuss below are invariant with respect to $\mathcal{A}_{s p}$, and relative invariants with respect to the full affine group.

In the case of Euclidean motions ( $A$ in (1) being a rotation matrix), it is well known that the Euclidean curvature $\kappa$ of a given plane curve is a differential invariant of the transformation. In the case of general affine transformations, in order to keep the invariance property, a new definition of curvature is necessary. In what follows, this affine curvature will be defined [8, 9, 18, 34]. See also [8,9] for extensive treatments of affine differential geometry.

Let $\mathcal{C}: S^{1} \rightarrow \mathbf{R}^{2}$ be an embedded curve with curve parameter $p$ (where $S^{1}$ denotes the unit circle). We assume throughout this section that all of our mappings are sufficiently smooth, so that all the relevant derivatives may be defined. A reparametrization of $C(p)$ to a new parameter $s$ can be performed such that

$$
\left[\mathcal{C}_{s}, \mathcal{C}_{s s}\right]=1
$$

where $[X, Y]$ stands for the determinant of the $2 \times 2$ matrix whose columns are given by the vectors $X, Y \in \mathbf{R}^{2}$. This relation is invariant under proper affine transformations, and the parameter $s$ is called the affine arc-length. Setting

$$
g(p):=\left[\mathcal{C}_{p}, \mathcal{C}_{p p}\right]^{1 / 3},
$$

the parameter $s$ is explicitly given by

$$
s(p)=\int_{0}^{p} g(\xi) d \xi
$$

Note that in the above standard definitions, we have assumed that $g$ (the affine metric) is different from zero at each point of the curve, i.e., the curve has no inflection points. Since we will be considering closed smooth curves for our evolutions, in utilizing this classical affine differential geometry, we must restrict ourselves to strictly convex curves $[8,9,18,30]$. In Section 4 we will show how to get around this problem for the affine evolution of non-convex curves.

It is easy to see that the following relations hold $[8,9,30]$ :

$$
\begin{gathered}
d s=g d p \\
\mathcal{C}_{s}=\mathcal{C}_{p} \frac{d p}{d s} \\
\mathcal{C}_{s s}=\mathcal{C}_{p p}\left(\frac{d p}{d s}\right)^{2}+\mathcal{C}_{p} \frac{d^{2} p}{d s^{2}} .
\end{gathered}
$$

$\mathcal{C}_{s}$ is called the affine tangent vector and $\mathcal{C}_{s s}$ the affine normal vector. 
By differentiating (2) we obtain

$$
\left[\mathcal{C}_{s}, \mathcal{C}_{s s s}\right]=0
$$

Hence, the two vectors $\mathcal{C}_{s}$ and $\mathcal{C}_{\text {sss }}$ are linearly dependent and so there exists $\mu$ such that

$$
\mathcal{C}_{s s s}+\mu \mathcal{C}_{s}=0
$$

The last equation implies

$$
\mu=\left[\mathcal{C}_{s s}, \mathcal{C}_{s s s}\right]
$$

and $\mu$ is called the affine curvature. The affine curvature is the simplest non-trivial differential affine invariant of the curve $\mathcal{C}[9]$. Note that $\mu$ can also be computed as

$$
\mu=\left[\mathcal{C}_{s s s s}, \mathcal{C}_{s}\right]
$$

For the exact expression of $\mu$ as a function of the original parameter $p$, see [9]. For proofs on the invariance property of the above defined affine concepts, see [30].

In the Euclidean case, constant Euclidean curvature $\kappa$ is obtained only for circular arcs and straight lines. Further, the Euclidean osculating figure of a curve $\mathcal{C}$ at a point $x$ is always the circle with radius $1 / \kappa(x)$ whose center lies on the normal at $x[34]$.

In the affine case, the conics (parabola, ellipse, and hyperbola) are the only curves with constant affine curvature $\mu(\mu=0, \mu>0$, and $\mu<0$, respectively). Therefore, the ellipse is the only closed curve with constant affine curvature. The affine osculating conic of a curve $\mathcal{C}$ at a non-inflection point $x$ is a parabola, ellipse, or hyperbola, depending on whether the affine curvature $\mu$ at $x$ is zero, positive, or negative $[9,18,34]$.

\section{AfFine SHORTENing OF CONVEX CURVES}

We present now the affine curve shortening flow or affine invariant geometric heat equation for convex curves as formulated in [30]. We will also summarize its relation with the classical Euclidean curve shortening flow, and recall its basic properties. For all the details and proofs, the reader is referred to [30].

Let $\mathcal{C}(p, t): S^{1} \times[0, T) \rightarrow \mathbf{R}^{2}$ be a family of embedded curves where $t$ parameterizes the family and $p$ parameterizes each curve. The classical Euclidean curve shortening flow or geometric heat equation is given by $[15,16]$

$$
\left\{\begin{aligned}
\frac{\partial \mathcal{C}(p, t)}{\partial t} & =\frac{\partial^{2} \mathcal{C}(p, t)}{\partial \tilde{s}^{2}}=\kappa(p, t) \mathcal{N} \\
\mathcal{C}(\cdot, 0) & =\mathcal{C}_{0}(\cdot)
\end{aligned}\right.
$$

where

$$
\tilde{s}=\int\left\|\mathcal{C}_{p}\right\| d p
$$

is the Euclidean arclength and $\kappa$ and $\mathcal{N}$ are the curvature and unit normal, respectively.

As pointed out in the Introduction, an embedded curve converges to a round point when evolving according to $(12)[15,16]$. If the initial curve is non-convex, it becomes convex before it shrinks to a point [16]. Other properties of this Euclidean curve shortening, and of Euclidean curve evolution in general, can be found for example in $[6,15,16,17,19,35]$. 
In [30], we argue that the affine analogue of (12) is given by

$$
\left\{\begin{aligned}
\frac{\partial \mathcal{C}(p, t)}{\partial t} & =\mathcal{C}_{s s}(p, t) \\
\mathcal{C}(\cdot, 0) & =\mathcal{C}_{0}(\cdot)
\end{aligned}\right.
$$

Based on this evolution, an affine invariant curve shortening theory is developed in [30] for a strictly convex initial curve $\mathcal{C}_{0}(\cdot)$. We now summarize those results which will be necessary for our discussion in the next section. All the proofs of the following results may be found in [30].

Lemma 3.1. The solution of the evolution (13) is an absolute invariant of the group $\mathcal{A}_{\text {sp }}$ of special affine motions, and a relative invariant of the group $\mathcal{A}$ of proper affine motions.

Theorem 3.2. Notation as above. Then $\mathcal{C}(p, t)$ evolving according to (13) remains convex.

Theorem 3.3. The solution of (13) exists as long as the area enclosed by the evolving curve is bounded away from zero.

Theorem 3.4. Any convex smooth embedded curve converges to an elliptical point when evolving according to (13). This convergence is in the sense that the family of dilated normalized curves converges in the Hausdorff metric to an ellipse.

We would like now to briefly note the connection of (13) with a gradient flow of the area. We follow here the treatment of [27] to which the reader is referred to for all of the details. Since affine geometry is defined only for convex curves [8], we will initially have to restrict ourselves to the (Fréchet) space of four times differentiable convex closed curves in the plane, i.e.,

$$
\mathbf{C}_{0}:=\left\{\mathcal{C}:[0,1] \rightarrow \mathbf{R}^{2}: \mathcal{C} \text { is convex, closed and } C^{4}\right\} .
$$

As above, let $d s$ denote the affine arc-length. Then, for $L_{a f f}:=\oint d s$ the affine length [8], we define on $\mathbf{C}_{0}$

$$
\|\mathcal{C}\|_{a f f}:=\int_{0}^{1}\|\mathcal{C}(p)\|_{a} d p=\int_{0}^{L_{a f f}}\|\mathcal{C}(s)\|_{a} d s
$$

where

$$
\|\mathcal{C}(p)\|_{a}:=\left[\mathcal{C}(p), \mathcal{C}_{p}(p)\right]
$$

Note that the area enclosed by $\mathcal{C}$ is just

$$
A=\frac{1}{2} \int_{0}^{1}\|\mathcal{C}(p)\|_{a} d p=\frac{1}{2} \int_{0}^{1}\left[\mathcal{C}, \mathcal{C}_{p}\right] d p=\frac{1}{2}\|\mathcal{C}\|_{a f f}
$$

Observe that

$$
\left\|\mathcal{C}_{s}\right\|_{a}=\left[\mathcal{C}_{s}, \mathcal{C}_{s s}\right]=1, \quad\left\|\mathcal{C}_{s s}\right\|_{a}=\left[\mathcal{C}_{s s}, \mathcal{C}_{s s s}\right]=\mu
$$

where $\mu$ is the affine curvature as above. This makes the affine norm $\|\cdot\|_{a f f}$ consistent with the properties of the Euclidean norm on curves relative to the Euclidean arc-length $d \tilde{s}$. (Here we have that $\left\|\mathcal{C}_{\tilde{s}}\right\|=1,\left\|\mathcal{C}_{\tilde{s} \tilde{s}}\right\|=\kappa$.)

Let $\mathcal{C}(p, t)$ be a family of curves in $\mathbf{C}_{0}$. A straightforward computation reveals that the first variation of the area functional

$$
A(t)=\frac{1}{2} \int_{0}^{1}\left[\mathcal{C}, \mathcal{C}_{p}\right] d p
$$


is

$$
A^{\prime}(t)=-\int_{0}^{L_{a f f}(t)}\left[\mathcal{C}_{t}, \mathcal{C}_{s}\right] d s .
$$

Therefore the gradient flow which will decrease the area as quickly as possible relative to $\|\cdot\|_{\text {aff }}$ is exactly $\mathcal{C}_{t}=\mathcal{C}_{s s}$, i.e., equation (13). In this sense then, (13) is an affine curve shortening flow.

\section{Extension of AFFine HeAt EQUATION TO NON-CONVEX CURVES}

Let $\mathcal{C}(p, t): S^{1} \times[0, T) \rightarrow \mathbf{R}^{2}$ be a family of curves where $t$ parametrizes the family and $p$ parametrizes each curve.

We now make the standard argument about how to drop the tangential component of the velocity vector in curve evolution problems such as (13). Accordingly, given a (parametrized) plane curve $\mathcal{C}(p, t)$, we denote its image by $\operatorname{Img}[\mathcal{C}(p, t)]$. Therefore, if the curve $\mathcal{C}(p, t)$ is parametrized by a new parameter $w$, such that $w=w(p, t), \partial w / \partial p>0$, we obtain

$$
\operatorname{Img}[\mathcal{C}(p, t)]=\operatorname{Img}[\mathcal{C}(w, t)] .
$$

The following lemma (whose proof may be found in [12] and also in [31]) refers to the image of a curve evolving with tangential velocity component. Let $\mathcal{T}$ and $\mathcal{N}$ be the Euclidean unit tangent and Euclidean unit normal of the curve, respectively. Then we have:

Lemma 4.1. Let $\beta$ be a geometric quantity for a curve, i.e., a function whose definition is independent of a particular parametrization. Then a family of curves which evolves according to

$$
\mathcal{C}_{t}=\alpha \mathcal{T}+\beta \mathcal{N}
$$

can be converted into the solution of

$$
\mathcal{C}_{t}=\bar{\alpha} \mathcal{T}+\bar{\beta} \mathcal{N}
$$

for any continuous function $\bar{\alpha}$, by changing the space parametrization of the original solution. Since $\beta$ is a geometric function, $\beta=\bar{\beta}$ when the same point in the (geometric) curve is taken.

In particular, the lemma shows that $\operatorname{Img}[\mathcal{C}(p, t)]=\operatorname{Img}[\hat{\mathcal{C}}(w, t)]$, where $\mathcal{C}(p, t)$ and $\hat{\mathcal{C}}(w, t)$ are the solutions of

$$
\mathcal{C}_{t}=\alpha \mathcal{T}+\beta \mathcal{N}
$$

and

$$
\hat{\mathcal{C}}_{t}=\bar{\beta} \mathcal{N}
$$

respectively.

Now in [30], it is noted that

$$
\mathcal{C}_{s s}=\kappa^{1 / 3} \mathcal{N}+\text { tangential component, }
$$

that is, the Euclidean normal component of the affine normal $\mathcal{C}_{s s}$ is equal to $\kappa^{1 / 3} \mathcal{N}$. Since $\kappa^{1 / 3}$ vanishes at the inflection points, by the above argument we see immediately that the affine invariant flow given by (13) is geometrically equivalent to

$$
\begin{aligned}
\mathcal{C}_{t} & =\kappa^{1 / 3} \mathcal{N}, \\
\mathcal{C}_{0}(\cdot) & =\mathcal{C}(\cdot, 0) .
\end{aligned}
$$


If $\mathcal{C}$ is the solution of (13) and $\hat{\mathcal{C}}$ is the solution of (15), then

$$
\operatorname{Img}[\mathcal{C}]=\operatorname{Img}[\hat{\mathcal{C}}] .
$$

From the above, $\operatorname{Img}[\hat{\mathcal{C}}]$ is an affine invariant of the evolution (15). Note that the image of the curve (i.e., the geometric curve) is the affine invariant, not the parametrized curve. However, this evolution is well-defined for non-convex curves. We will refer to (15) as the affine invariant geometric heat equation.

Hence, in spite of the non-existence of the classical affine differential invariants for non-convex curves $[8,18]$, our above analysis makes it possible to extend the affine evolutionary flow (13) to the non-convex case. This is due to the invariant property of the inflection points, and the possibility to "ignore" the tangential velocity component since this does not effect the geometric evolution of the flow. See also $[1,2]$ for an equivalent formulation of (15) studied from a viscosity solution framework.

Remark. In $[24,25,26]$ we classify invariant flows under a given Lie group action. Using this classification one may show in particular that (15) is the simplest (in the sense of having the smallest number of spatial derivatives) non-trivial planar evolution equation invariant under the special affine group, and thus is unique (up to constant factor).

We will devote the remainder of this paper to the study the regularity properties of (15).

\section{Short-time EXISTEnCE}

In this section, we verify the short-time existence for the flow (15). Because the conditions of Angenent $[4,5]$ do not apply to the function $\kappa^{1 / 3}$, we must go through an approximation argument. We assume that our initial curve $\mathcal{C}_{0}(\cdot)$ is $C^{2}$.

We first approximate $\kappa^{1 / 3}$ by $v=\phi(\kappa)=\phi_{\delta}(\kappa)$, where

$$
\phi_{\delta}(\kappa)=\frac{1}{3} \int_{0}^{\kappa} \frac{d s}{\sqrt[3]{\delta+s^{2}}} .
$$

Note that $\phi^{\prime}(\kappa)>0$, and $\phi \in C^{\infty}(\mathbf{R})$. Assume that $\left\{\mathcal{C}_{t}: 0<t<T\right\}$ evolves by $v=\phi(\kappa)$ with $|\kappa| \leq M$ for $0<t<T$. Using Nash-Moser iteration, we will derive an upper bound for

$$
w:=\frac{\partial v}{\partial \tilde{s}} .
$$

Recall that $\tilde{s}$ denotes the Euclidean arc-length.

We begin with the evolution equations for $v$ and $w$. From

$$
\frac{\partial \kappa}{\partial t}=v_{\tilde{s} \tilde{s}}+\kappa^{2} v
$$

and $v=\phi(\kappa)$ we get that

$$
\frac{\partial v}{\partial t}=\phi^{\prime}(\kappa) v_{\tilde{s} \tilde{s}}+\kappa^{2} v \phi^{\prime}(\kappa) .
$$

Using the commutation relation

$$
\left[\frac{\partial}{\partial t}, \frac{\partial}{\partial \tilde{s}}\right]=\kappa v \frac{\partial}{\partial \tilde{s}},
$$


we get the evolution of $w$ :

$$
\begin{aligned}
\frac{\partial w}{\partial t} & =\frac{\partial}{\partial \tilde{s}}\left(\frac{\partial v}{\partial t}\right)+\kappa v w \\
& =\left(\phi^{\prime}(\kappa) w_{\tilde{s}}\right)_{\tilde{s}}+\left(\kappa^{2} v \phi^{\prime}(\kappa)\right)_{\tilde{s}}+\kappa v w .
\end{aligned}
$$

\subsection{Nash-Moser iteration applied to $w$. Set}

$$
X_{p}(t):=\int_{\mathcal{C}_{t}}|w|^{p} d \tilde{s}
$$

Then

$$
\begin{aligned}
X_{p}^{\prime}(t) & =\int_{\mathcal{C}_{t}}\left(p|w|^{p-1} w_{t}-|w|^{p} \kappa v\right) d \tilde{s} \\
& =\int_{\mathcal{C}_{t}}\left\{p|w|^{p-1}\left(\phi^{\prime}(\kappa) w_{\tilde{s}}+\kappa^{2} v \phi^{\prime}(\kappa)\right)_{\tilde{s}}+p \kappa v|w|^{p}-\kappa v|w|^{p}\right\} d \tilde{s}
\end{aligned}
$$

Integrating by parts, and using $2\left|\kappa^{2} w_{\tilde{s}}\right| \leq \kappa^{4}+w_{\tilde{s}}^{2}$, we get

$$
\begin{aligned}
X_{p}^{\prime}(t)= & -p(p-1) \int_{\mathcal{C}_{t}} \phi^{\prime}(\kappa)\left\{|w|^{p-2} w_{\tilde{s}}^{2}+\kappa^{2}|w|^{p-2} w_{\tilde{s}}\right\} d \tilde{s}+(p-1) \int_{\mathcal{C}_{t}} \kappa v|w|^{p} d \tilde{s} \\
\leq & -\frac{p(p-1)}{2} \int_{\mathcal{C}_{t}} \phi^{\prime}(\kappa)|w|^{p-2} w_{\tilde{s}}^{2} d \tilde{s}+\frac{p(p-1)}{2} \int_{\mathcal{C}_{t}} \kappa^{4} \phi^{\prime}(\kappa)|w|^{p-2} d \tilde{s} \\
& \quad+(p-1) \int_{\mathcal{C}_{t}} \kappa v|w|^{p} d \tilde{s} \\
\leq & -2(1-1 / p) \int_{\mathcal{C}_{t}} \phi^{\prime}(\kappa)\left(\left(w^{p / 2}\right)_{\tilde{s}}\right)^{2} d \tilde{s}+\frac{p^{2}}{2} \int_{\mathcal{C}_{t}} \kappa^{4} \phi^{\prime}(\kappa)|w|^{p-2} d \tilde{s} \\
& +p \int_{\mathcal{C}_{t}} \kappa v|w|^{p} d \tilde{s} .
\end{aligned}
$$

We now take $p \geq 2$, so that $1-1 / p \geq 1 / 2$. Let $\sup |\kappa|=M$ and $\inf \phi^{\prime}(\kappa)=\hat{\delta}$. Then we can find an approximating $\phi(\kappa)$ such that

$$
\left|\kappa^{4} \phi^{\prime}(\kappa)\right|+|\kappa \phi(\kappa)| \leq C,
$$

for some constant $C$ uniformly in the approximating $\phi(\kappa)$. Then we get

$$
X_{p}^{\prime}(t) \leq-\hat{\delta} \int_{\mathcal{C}_{t}}\left(\left(w^{p / 2}\right)_{\tilde{s}}\right)^{2} d \tilde{s}+\frac{C}{2} p^{2} X_{p-2}(t)+C p X_{p}(t)
$$

We want to get rid of the first term of (20) by means of an interpolation inequality.

Proposition 5.1. If $f$ is a function on $\mathcal{C}_{t}$ with $f \in C^{1}$, and if there exists a point $x_{0} \in \mathcal{C}_{t}$ with $f\left(x_{0}\right)=0$, then

$$
\sup _{\mathcal{C}_{t}} f^{2} \leq\|f\|_{2}\left\|f^{\prime}\right\|_{2} .
$$


Proof. We integrate $\left(f^{2}\right)_{\tilde{s}}=2 f f^{\prime}$ from the zero $x_{0}$ to any other point $x \in \mathcal{C}_{t}$ along two different paths $\gamma_{1}$ and $\gamma_{2}$ from $x_{0}$ to $x$ :

$$
\begin{aligned}
f(x)^{2} & =\int_{\gamma_{1}} 2 f f^{\prime} d \tilde{s}=\int_{\gamma_{2}} 2 f f^{\prime} d \tilde{s} \\
& =\int_{\gamma_{1}} f f^{\prime} d \tilde{s}+\int_{\gamma_{2}} f f^{\prime} d \tilde{s} \\
& \leq \int_{\mathcal{C}_{t}}\left|f f^{\prime}\right| d \tilde{s} \\
& \leq\|f\|_{2} \cdot\left\|f^{\prime}\right\|_{2} \quad \text { by Cauchy-Schwarz, }
\end{aligned}
$$

which completes the proof.

Apply this to $f=w^{p / 2}$. Since $w=v_{\tilde{s}}$ and $v$ must attain a maximum, there must be a point where $f=\left(v_{\tilde{s}}\right)^{p / 2}=0$. We get

$$
\sup |w|^{p} \leq\left(\int_{\mathcal{C}_{t}}|w|^{p} d \tilde{s}\right)^{1 / 2}\left(\int_{\mathcal{C}_{t}}\left(w^{p / 2}\right)_{\tilde{s}} d \tilde{s}\right)^{1 / 2}
$$

But since

we obtain

$$
\left(\int_{\mathcal{C}_{t}} w^{p} d \tilde{s}\right) \leq \sup |w|^{p / 2}\left(\int_{\mathcal{C}_{t}}|w|^{p / 2} d \tilde{s}\right)
$$

$$
\begin{aligned}
\left(\int_{\mathcal{C}_{t}}|w|^{p} d \tilde{s}\right) & \leq\left(\int_{\mathcal{C}_{t}} w^{p} d \tilde{s}\right)^{1 / 4}\left(\int_{\mathcal{C}_{t}}\left(w^{p / 2}\right)_{\tilde{s}}^{2} d \tilde{s}\right)^{1 / 4}\left(\int_{\mathcal{C}_{t}}|w|^{p / 2} d \tilde{s}\right) \\
& \leq\left(\int_{\mathcal{C}_{t}}\left(w^{p / 2}\right)_{\tilde{s}}^{2} d \tilde{s}\right)^{1 / 3}\left(\int_{\mathcal{C}_{t}}|w|^{p / 2} d \tilde{s}\right)^{4 / 3},
\end{aligned}
$$

and hence,

$$
-\int_{\mathcal{C}_{t}}\left(w^{p / 2}\right)_{\tilde{s}}^{2} d \tilde{s} \leq-\frac{X_{p}^{3}}{X_{p / 2}^{4}} .
$$

Substituting in (20), we find that

$$
X_{p}^{\prime}(t) \leq-\hat{\delta} \frac{X_{p}^{3}}{X_{p / 2}^{4}}+\frac{C p^{2}}{2} X_{p-2}+C p X_{p}
$$

Next, if we use the inequality

$$
X_{p-2}=\int_{\mathcal{C}_{t}}|w|^{p-2} d \tilde{s} \leq \int_{\mathcal{C}_{t}}\left(1+|w|^{p}\right) d \tilde{s} \leq L+X_{p}
$$

(where $L=L\left(\mathcal{C}_{t}\right)$ is the Euclidean length of $\mathcal{C}_{t}$ ), we get that

$$
X_{p}^{\prime}(t) \leq-\hat{\delta} \frac{X_{p}^{3}}{X_{p / 2}^{4}}+C\left(\frac{p^{2}+2 p}{2}\right) X_{p}+\frac{C L p^{2}}{2},
$$

which holds for $p \geq 2$.

The differential inequality (22) allows us to turn bounds for $X_{p / 2}$ into bounds for $X_{p}$. We must begin with $p=2$, in which case we have no bound on

$$
X_{1}=X_{p / 2}=\int_{\mathcal{C}_{t}}|w| d \tilde{s}=\int_{\mathcal{C}_{t}}\left|v_{\tilde{s}}\right| d \tilde{s} .
$$


But, for $p=2$ we have the bound

$$
\begin{aligned}
\left\|v_{\tilde{s}}\right\|_{2} & \leq\|v\|_{2}^{1 / 2} \cdot\left\|v_{\tilde{s} \tilde{s}}\right\|_{2}^{1 / 2} \\
& \leq \sup |v|^{1 / 2} \cdot L\left(\mathcal{C}_{t}\right)^{1 / 2}\left\|v_{\tilde{s} \tilde{s}}\right\|_{2}^{1 / 2} .
\end{aligned}
$$

Since $v=\phi(\kappa)$ and $L\left(\mathcal{C}_{t}\right)$ are uniformly bounded, we get that

$$
\|w\|_{2} \leq C_{1}\left\|w_{\tilde{s}}\right\|_{2}^{1 / 2}, \text { for } C_{1} \text { constant, }
$$

and hence from (20), (22), (23),

$$
\begin{aligned}
\frac{d X_{2}}{d t} & \leq-\hat{\delta} \int_{\mathcal{C}_{t}}\left(w_{\tilde{s}}\right)^{2} d \tilde{s}+4 C X_{2}+2 C L \\
& \leq-\frac{\hat{\delta}}{C_{1}^{4}}\left(X_{2}\right)^{2}+4 C X_{2}+2 C L
\end{aligned}
$$

which implies

$$
X_{2}(t) \leq \frac{A_{2}}{t} \quad(0<t<T)
$$

for a sufficiently large constant $A_{2}$ depending only on $\sup |\kappa|=M$, inf $\phi^{\prime}=$ $\hat{\delta}, \sup L\left(\mathcal{C}_{t}\right)$, and $T$.

5.2. The iteration step. Suppose we have for some $p \geq 2$

$$
\|w\|_{p / 2} \leq \frac{A}{t^{\alpha}}
$$

or equivalently

$$
X_{p / 2}(t) \leq A^{p / 2} t^{-p \alpha / 2} .
$$

Then (22) implies for $X(t)=X_{p}(t)$ the differential inequality

$$
\frac{d X}{d t} \leq \frac{-\hat{\delta}}{A^{2 p}} t^{2 \alpha p} X^{3}(t)+C^{\prime}\left(p^{2}+2 p\right) X(t)+C^{\prime \prime} p^{2} \quad(0<t<T),
$$

where $C^{\prime}:=\frac{C}{2}$ and $C^{\prime \prime}:=\frac{C L}{2}$.

We now try to find a supersolution of the form

$$
\hat{X}(t)=B^{p} t^{-p \beta}
$$

for (25), i.e., $\hat{X}(t)$ is required to satisfy the reverse inequality. Assume that $B \geq 2$. Substituting, we see that we want (for $0<t<T$ )

$$
-p \beta B^{p} t^{-1-p \beta} \geq \frac{-\hat{\delta}}{A^{2 p}} t^{2 \alpha p-3 \beta p} B^{3 p}+C^{\prime}\left(p^{2}+2 p\right) B^{p} t^{-p \beta}+C^{\prime \prime} p^{2},
$$

or equivalently,

$$
-p \beta \geq-\hat{\delta}\left(\frac{B}{A}\right)^{2 p} t^{1+2 p(\alpha-\beta)}+C^{\prime}\left(p^{2}+2 p\right) t+C^{\prime \prime} p^{2} B^{-p} t^{1+p \beta} .
$$

Choose

$$
\beta:=\alpha+\frac{1}{2 p}
$$

and note that since $B \geq 2$, we have that $C^{\prime \prime} p^{2} B^{-p} \leq \bar{C}(\bar{C}$ a constant), and so we require that

$$
-p \beta \geq-\hat{\delta}\left(\frac{B}{A}\right)^{2 p}+C^{\prime}\left(p^{2}+2 p\right) T+\bar{C} T^{3 / 2+p \alpha},
$$


which holds if we take

$$
B=A\left(\frac{p \beta+\bar{C}_{1}\left(p^{2}+2 p\right)+\bar{C}_{2} T^{p \alpha}}{\hat{\delta}}\right)^{\frac{1}{2 p}},
$$

where

$$
\bar{C}_{1}:=C^{\prime} T, \quad \bar{C}_{2}:=\bar{C} T^{3 / 2} .
$$

Recapping, we have shown that if $\|w\|_{p / 2} \leq A t^{-\alpha}$ for $0<t<T$, then $\|w\|_{p} \leq B t^{-\beta}$, i.e.,

$$
\|w\|_{p} \leq A\left(\frac{p \beta+\bar{C}_{1}\left(p^{2}+2 p\right)+\bar{C}_{2} T^{p \alpha}}{\hat{\delta}}\right)^{\frac{1}{2 p}} t^{-\alpha-\frac{1}{2 p}}, \quad 0<t<T .
$$

We can now carry out the iteration. Let

$$
\begin{aligned}
& p_{k}=2^{k+1}, \quad k \geq 0, \\
& \alpha_{k}=\frac{1}{2}+\sum_{j=3}^{k+2} 2^{-j}, \quad k \geq 1, \\
& \alpha_{0}=\frac{1}{2} .
\end{aligned}
$$

Let $A_{0}$ be the constant from estimate (24), and define

$$
A_{k+1}:=A_{k}\left(\frac{p_{k} \beta+\bar{C}_{1}\left(p_{k}^{2}+p_{k}\right)+\bar{C}_{2} T^{p_{k} \alpha_{k}}}{\hat{\delta}}\right)^{\frac{1}{p_{k+1}}} .
$$

Then

$$
\lim _{k \rightarrow \infty} A_{k}=A_{\infty}<\infty, \quad \lim _{k \rightarrow \infty} \alpha_{k}=\frac{3}{4}
$$

and so we see that

$$
\|w\|_{\infty} \leq A_{\infty} t^{-\frac{3}{4}} \text { for } 0<t<T .
$$

Note that the constant $A_{\infty}$ only depends on $\hat{\delta}, T$, sup $|\kappa|=M$, the length of $\mathcal{C}_{t}$ $(L)$, and

$$
\sup _{|\kappa| \leq M}\left\{\kappa^{4}\left|\phi^{\prime}(\kappa)\right|+\kappa|\phi(\kappa)|\right\}
$$

We now can prove the following result:

Theorem 5.2. Given $\mathcal{C}_{0}(\cdot) \in C^{2}$, there exists a (classical) solution of $(15)\{\mathcal{C}(\tilde{s}, t)$ : $0<t<T\}$ with

as $t \uparrow T$.

$$
\max _{\mathcal{C}_{t}}|\kappa|=\kappa(t) \uparrow \infty
$$

Proof. As above, we set

$$
v=\phi_{\delta}(\kappa):=\frac{1}{3} \int_{0}^{\kappa}\left(\delta+s^{2}\right)^{-1 / 3} d s .
$$

We assume that $\mathcal{C}_{t}^{\delta}$ evolves according to

$$
\frac{\partial \mathcal{C}}{\partial t}=\phi_{\delta}(\kappa) \mathcal{N} .
$$

Then as in $(17,18,19)$, we get that

$$
\frac{\partial \kappa}{\partial t}=v_{\tilde{s} \tilde{s}}+\kappa^{2} v
$$




$$
\begin{gathered}
\frac{\partial v}{\partial t}=\phi_{\delta}^{\prime}(\kappa) v_{\tilde{s} \tilde{s}}+\kappa^{2} v \phi_{\delta}^{\prime}(\kappa) \\
\frac{\partial w}{\partial t}=\left(\phi_{\delta}^{\prime}(\kappa) w_{\tilde{s}}\right)_{\tilde{s}}+\left(\kappa^{2} v \phi_{\delta}^{\prime}(\kappa)\right)_{\tilde{s}}+\kappa v w,
\end{gathered}
$$

where as above $w:=v_{\tilde{s}}$.

Now equation (26) implies that

$$
\frac{d}{d t} \sup |\kappa| \leq(\sup |\kappa|)^{2} \phi_{\delta}(\sup |\kappa|)
$$

Hence if $\Phi(t, A)$ is the solution of

$$
\begin{gathered}
\frac{\partial \Phi_{\delta}}{\partial \tau}(\tau, A)=\phi_{\delta}\left(\Phi_{\delta}(\tau, A)\right) \Phi_{\delta}(\tau, A)^{2} \\
\Phi_{\delta}(0, A)=A
\end{gathered}
$$

then

$$
\sup _{\mathcal{C}_{t}^{\delta}}|\kappa| \leq \Phi_{\delta}\left(t, \sup _{\mathcal{C}_{0}^{\delta}}|\kappa|\right) \text {. }
$$

Using the Nash-Moser iteration argument as above, we get that

$$
\sup _{\mathcal{C}_{t}}|w| \leq \frac{C}{t^{3 / 4}}, \quad 0<t<T,
$$

where $C$ depends on

$$
\sup _{0<t<T} \sup _{\mathcal{C}_{t}}|\kappa|
$$

but not on $\delta$.

The latter bounds then imply the existence of a solution as long as sup $|\kappa|$ remains bounded. We are almost done now. Indeed, we evolve $\mathcal{C}_{0}^{\delta}$ by $v=\phi_{\delta}(\kappa)$, with the resulting family $\left\{\mathcal{C}_{t}^{\delta}: 0<t<T\right\}$ for some $T$ independent of $\delta$. Our preceding estimates imply that we can extract a subsequence $\delta_{i} \downarrow 0$ on which $v$ and $\kappa$ converge uniformly, and still retain the estimate (30). The limit is the required family satisfying the conclusion of Theorem 5.2.

Remark. The bound on $w=v_{\tilde{s}}$ implies that $\kappa^{1 / 3}(t)$ is Lipschitz on $\mathcal{C}_{t}$, and so inflection points have at least fifth order contact with their tangents. Recall that for a generic $C^{3}$ curve, one only has third order contact at inflection points.

\section{Uniqueness of SOLUTION With $C^{2}$ InItIAL DATA}

In this section, we give a uniqueness result for solutions of the affine heat equation. Accordingly, we state,

Theorem 6.1. Let $\mathcal{C}_{0}$ be a $C^{2}$ curve. Then there is a unique classical solution $\left\{\mathcal{C}_{t}: 0<t<T\right\}$ of $(15)$.

Proof. Set, for $\varepsilon$,

$$
\mathcal{C}_{0}^{\varepsilon}(\tilde{s})=\mathcal{C}_{0}(\tilde{s})+\varepsilon \mathcal{N}_{0}(\tilde{s}),
$$

where $\mathcal{N}_{0}(\tilde{s})$ is the unit normal to $\mathcal{C}_{0}(\tilde{s})$. Using the above notation, for $\delta>0$ we evolve $\mathcal{C}_{0}^{\varepsilon}(\tilde{s})$ by $v=\phi_{\delta}(\kappa)$. We thereby obtain a family of classical solutions

$$
\left\{\mathcal{C}_{t}^{\varepsilon, \delta}: 0<t<T\right\}
$$


on some (short) time interval. Denote the normal variation with respect to $\varepsilon$ by $h=h^{\varepsilon, \delta}(\tilde{s}, t)$, i.e.,

$$
h^{\varepsilon, \delta}(\tilde{s}, t)=\left\langle\frac{\partial \mathcal{C}^{\varepsilon, \delta}(\tilde{s}, t)}{\partial \varepsilon}, \mathcal{N}^{\varepsilon, \delta}(\tilde{s}, t)\right\rangle
$$

where $\mathcal{N}^{\varepsilon, \delta}(\tilde{s}, t)$ is the unit normal to $\mathcal{C}^{\varepsilon, \delta}(\tilde{s}, t)$. The normal variation $h$ evolves by the same equation as the normal velocity:

$$
\frac{\partial h}{\partial t}=\phi_{\delta}^{\prime}(\kappa) \frac{\partial^{2} h}{\partial \tilde{s}^{2}}+\phi_{\delta}^{\prime}(\kappa) \kappa^{2} h .
$$

By the argument as in Section 5 for $w=v_{\tilde{s}}$, we get that $\left|\frac{\partial h}{\partial \tilde{s}}\right|$ is uniformly bounded. Taking $|\varepsilon|<\varepsilon_{0}$ for some $\varepsilon_{0}>0$, we can assume that $\left.h\right|_{t=0} \equiv 1$. (Note that at $t=0$, $\mathcal{C}_{0}^{\varepsilon, \delta}$ does not depend on $\delta$. For $\left.h\right|_{t=0} \equiv 1$, the curves $\mathcal{C}^{\varepsilon}$ are basically "Huygens wave fronts" generated by $\mathcal{C}^{\varepsilon=0}$.) By the maximum principle applied to (31), it then follows that

$$
\sup _{\varepsilon} \sup _{0<t<T} \sup _{\mathcal{C}_{t}^{\varepsilon, \delta}} h^{\varepsilon, \delta}<C
$$

for some constant $C$. Hence the curves $\mathcal{C}_{t}^{\varepsilon, \delta}$ depend in a $C^{1}$ manner on $\varepsilon$, uniformly in $\delta, t$. So one can take a subsequence $\delta_{i} \downarrow 0$ for which the

$$
\mathcal{C}_{t}^{\varepsilon, \delta} \rightarrow \mathcal{C}_{t}^{\varepsilon}
$$

where the $\mathcal{C}_{t}^{\varepsilon}$ also depend $C^{1}$ on $\varepsilon$.

This construction provides us with two classical solutions of the evolution equation (15) with velocity $\kappa^{1 / 3}$, namely $\left\{\mathcal{C}_{t}^{\varepsilon}, \mathcal{C}_{t}^{-\varepsilon}\right\}$, between which any other classical solution must lie (maximum principle--see the next section). But our sup $|h|$ estimate (32) shows that the width of the region between $\mathcal{C}_{t}^{\varepsilon}$ and $\mathcal{C}_{t}^{-\varepsilon}$ is $O(\varepsilon)$. Since $\varepsilon$ is arbitrary, the result follows.

\section{MAXIMUM PRINCIPLES}

Let the graph of $y=u(x, t)$ evolve by $v=\kappa^{1 / 3}$. Then it is easy to compute $[24,25]$ that $u$ satisfies the local affine heat equation

$$
u_{t}=\left(u_{x x}\right)^{1 / 3} \text {. }
$$

In this section we consider two solutions $u$ and $\bar{u}$ of (33) on a rectangle $Q=$ $[a, b] \times\left[t_{0}, t_{1}\right]$. As always, the parabolic boundary of $Q$ is the union of the bottom $[a, b] \times\left\{t_{0}\right\}$ and the two sides $\{a\} \times\left[t_{0}, t_{1}\right]$ and $\{b\} \times\left[t_{0}, t_{1}\right]$ of $Q$.

Lemma 7.1 (Weak and Strong Maximum Principles). Assume $u$ and $\bar{u}$ are solutions of (33) on $Q$, and assume that $u, u_{x}, u_{x x}, u_{t}$ and the corresponding derivatives for $\bar{u}$ are continuous on $Q$.

(1) If $u \leq \bar{u}$ on the parabolic boundary of $Q$, then $u \leq \bar{u}$ on all of $Q$.

(2) If, in addition, $u<\bar{u}$ on one of the sides, then $u<\bar{u}$ in the interior of $Q$.

We remark that the stronger statement (2) in this lemma differs from the usual maximum principle in that we require $u<\bar{u}$ on an entire side of $Q$. The dichotomy "either $u \equiv \bar{u}$ or else $u<\bar{u}$ in the interior of $Q$ " which the usual strong maximum principle states does not hold for affine invariant heat equation (15). See Subsection 10.1 for some relevant examples. 
Proof. The first part follows from the standard maximum principle arguments. Let $m(t)$ be $\max \{\{0\} \cup\{u(x, t)-\bar{u}(x, t) \mid a \leq x \leq b\}\}$. Then $m(t)$ is continuous and equation (33) implies that the righthand derivative of $m(t)$ is non-negative. At $t=t_{0}$ we have $m\left(t_{0}\right)=0$, so we must have $m(t) \equiv 0$.

To prove the second part, we consider the difference $w(x, t)=\bar{u}(x, t)-u(x, t)$. Assume that $w \geq 0$ on the parabolic boundary, and moreover $w(a, t) \geq \delta$ for some constant $\delta>0$. The mean value theorem implies that $w$ satisfies

$$
w_{t}=M(x, t) w_{x x},
$$

except at points where both $u_{x x}$ and $\bar{u}_{x x}$ vanish. In fact,

$$
M(x, t)=\left(u(x, t)^{2 / 3}-u(x, t)^{1 / 3} \bar{u}(x, t)^{1 / 3}+\bar{u}(x, t)^{2 / 3}\right)^{-1}
$$

is uniformly bounded from below by some constant, say, $M(x, t) \geq M_{*}>0$.

Let $w_{\varepsilon}(x, t)$ be the solution of $w_{t}=\frac{1}{2} M_{*} w_{x x}$ on $Q$ with zero initial data, $w_{\varepsilon}(b, t) \equiv-\varepsilon$ and $w_{\varepsilon}(a, t) \equiv \delta$ for $t_{0}<t \leq t_{1}$. One easily verifies that $w_{\varepsilon, x x}>0$ in the interior of $Q$, e.g., by using the explicit formula for the solution.

Then we claim that $w>w_{\varepsilon}$ throughout $Q$. This is certainly true on the parabolic boundary where strict inequality even holds. Using $M \geq M_{*}$ and the convexity in $x$ of $w_{\varepsilon}$ one checks that $w_{\varepsilon}$ is a strict subsolution of (34), at any point where $M$ is defined. Now let $t_{*}$ be the supremum of all $t^{\prime} \leq t_{1}$ for which $w>w_{\varepsilon}$ for $t \leq t^{\prime}$ and all $x \in[a, b]$. Suppose that $t_{*}<t_{1}$. Then there would be some $x_{*} \in(a, b)$ at which

$$
\left\{\begin{array}{c}
w\left(x_{*}, t_{*}\right)=w_{\varepsilon}\left(x_{*}, t_{*}\right), \quad w_{x}\left(x_{*}, t_{*}\right)=w_{\varepsilon, x}\left(x_{*}, t_{*}\right), \\
\text { and } w_{t}\left(x_{*}, t_{*}\right) \leq w_{\varepsilon, t}\left(x_{*}, t_{*}\right)
\end{array}\right.
$$

and also

$$
w_{x x}\left(x_{*}, t_{*}\right) \geq w_{\varepsilon, x x}\left(x_{*}, t_{*}\right)>0 .
$$

This last inequality shows that $u_{x x}$ and $\bar{u}_{x x}$ cannot both vanish at $\left(x_{*}, t_{*}\right)$ so that $M$ is well defined at $\left(x_{*}, t_{*}\right)$, and $w$ satisfies (34) there. We can then apply the standard maximum principle argument to conclude that $w_{t}\left(x_{*}, t_{*}\right)>w_{\varepsilon, t}\left(x_{*}, t_{*}\right)$ and hence contradict (35).

We have shown $w \geq w_{\varepsilon}$ for arbitrary $\varepsilon>0$, and therefore also have $w \geq w_{0}$ on $Q$. Since $w_{0}>0$ in the interior of $Q$, this completes the proof.

\section{LOCAL CURVATURE BOUNDS}

In this section, we derive the following local estimate which will be useful in the sequel.

Theorem 8.1. Let $u(x, t)$ be a classical solution of (33) on $a \leq x \leq b, 0<t<T$, with

$$
M_{1}:=\sup _{x, t}\left\{\left|u_{x}\right|: a \leq x \leq b, 0<t<T\right\}<\infty .
$$

Then for any $\delta>0$ such that $a+\delta<b-\delta$, there exists a constant $C>0$ such that

$$
\left|u_{x x}(x, t)\right| \leq \frac{C}{t^{3 / 4}}
$$

for $a+\delta \leq x \leq b-\delta, \quad 0<t<T$. The constant $C$ only depends on $\delta$ and $M_{1}$.

Proof. The proof follows that of [5], Theorem 3.1. By comparing the graph with shrinking ellipses one finds that the bound for $\left|u_{x}\right|$ implies an $L^{\infty}$ bound for $u$ on 
any smaller interval $a+\delta \leq x \leq b-\delta, \quad 0 \leq t<T$. Indeed, $\left|u_{x}\right| \leq M_{1}$ implies that $|u(x, 0)| \leq M_{0}$ for some $M_{0}<\infty$. Now let $x_{0} \in(a, b)$ be given and set

$$
\delta=\min \left(x_{0}-a, b-x_{0}\right) .
$$

The ellipsoid with center at $\left(x_{0}, M_{0}+h\right)$, horizontal axis $\delta$, and vertical axis $h$, will evolve by $v=\kappa^{1 / 3}$ to its center in time $t=3 / 4(h \delta)^{2 / 3}$. As it shrinks to its center, it must remain disjoint from the graph $y=u(x, t)$, and thus we get an upper bound for $u$ at time $t$

$$
u(x, t) \leq M_{0}+h=M_{0}+\left(\frac{4 t}{3}\right)^{\frac{3}{2}} \frac{1}{\delta} .
$$

The same bound of course also applies to $-u(x, t)$.

Now, using the notation of [5], instead of $w^{\alpha, \xi}(x, t)$, we use the explicit (parabola) solutions of the local affine heat equation (33)

$$
w(x, t)=-\frac{1}{2}\left(\frac{x-\xi}{\varepsilon}\right)^{2}-\frac{t}{\varepsilon^{2 / 3}}+\alpha,
$$

and count intersections. (These are the affine "grim reapers". See [16].) The solutions (37) allow a slight simplification of the proof in [5]. We will sketch the argument for completeness, only giving the details where there is a modification in the original proof of Theorem 3.1.

Fix $\left(x_{0}, t_{0}\right)$. Then in order to estimate $u_{x x}\left(x_{0}, t_{0}\right)$, choose $\xi, \varepsilon, \alpha$ so that

$$
\begin{aligned}
u\left(x_{0}, t_{0}\right) & =w\left(\xi, \varepsilon, \alpha ; x_{0}, t_{0}\right), \\
u_{x}\left(x_{0}, t_{0}\right) & =w_{x}\left(\xi, \varepsilon, \alpha ; x_{0}, t_{0}\right) .
\end{aligned}
$$

The latter equations give one the freedom to choose $\varepsilon$ as small as one likes (and then $\xi, \alpha$ depend on $\varepsilon$ ).

Accordingly, we choose $\varepsilon>0$ so small such that at $t=0$,

$$
\left|w_{x}(\xi, \varepsilon, \alpha ; x, 0)\right|>M_{1}
$$

whenever

$$
|w(\xi, \varepsilon, \alpha ; x, 0)| \leq M_{1}
$$

i.e., we take a very narrow parabola. We also require that

$$
w(\xi, \varepsilon, \alpha ; x, 0)<-M_{1} \text { for } x \leq a, x \geq b .
$$

These conditions then imply that $u(\cdot, 0)-w(\cdot, 0)$ has exactly two simple zeros on $[a, b]$, and that

$$
u(a, t)-w(a, t)>0 \text { and } u(b, t)-w(b, t)>0
$$

hold for $0 \leq t \leq t_{0}$. Thus the function $u(\cdot, 0)-w(\cdot, 0)$ has two sign changes, and $u(x, t)-w(x, t)$ does not change sign on $\{a, b\}$ as $t$ increases.

We would like to apply the Sturmian theorem from [7] at this point. Indeed, if it were applicable, it would imply that $u\left(\cdot, t_{0}\right)-w\left(\cdot, t_{0}\right)$ has $\leq 2$ zeroes. Due to the degeneracy of the equation (33), we cannot apply the Sturmian theorem. However, Matano's elegant argument [21] can be applied since it only uses the weak maximum principle. (See our discussion in Section 10 below.) From this, we can conclude that $u\left(\cdot, t_{0}\right)-w\left(\cdot, t_{0}\right)$ has at most two sign changes.

The proof now goes exactly as in [5]. Indeed, the assumption that $u_{x x}\left(x_{0}, t_{0}\right)<$ $w_{x x}\left(x_{0}, t_{0}\right)$ leads to a contradiction, so we find

$$
u_{x x}\left(x_{0}, t_{0}\right) \geq w_{x x}\left(x_{0}, t_{0}\right)=-\varepsilon^{-2} .
$$


An upper bound is found in the same way by applying the above argument to $-u$ instead of $u$. Thus we have a pointwise estimate for $\left|u_{x x}\right|$, which upon computing the proper $\varepsilon$, turns out to be the stated estimate. Indeed, the exponent $3 / 4$ in (36) follows from scaling. For if $u_{t}=\left(u_{x x}\right)^{1 / 3}$, then

$$
\bar{u}(x, t)=\frac{1}{\lambda} u\left(\lambda x, \lambda^{4 / 3} t\right)
$$

also satisfies $\bar{u}_{t}=\left(\bar{u}_{x x}\right)^{1 / 3}$ with the same derivative bound.

\section{CONVEX AND CONCAVE ARCS}

In this section, we study the behavior of convex and concave arcs of a given curve under the affine evolution (15).

Let $X: S^{1} \times[0, T) \rightarrow \mathbf{R}^{2}$ be a normal parametrization of a solution $\left\{\mathcal{C}_{t}: 0 \leq\right.$ $t<T\}$ of $v=\kappa^{1 / 3}$. A convex (concave) arc $\beta \subset \mathcal{C}_{t}$ is the image of a maximal interval $[a, b] \subset \mathbf{R}$ on which $\kappa(\cdot, t) \geq 0$ (or $\kappa \leq 0$, respectively). (Note that we can regard $X(\cdot, t)$ as a periodic function defined on $\mathbf{R}$ in the usual way for each fixed $t \in[0, T)$.)

On the set $\{(p, t): \kappa(p, t) \neq 0\}$ we know that $\kappa$ is a solution of

$$
\kappa_{t}=\phi(\kappa)_{\tilde{s} \tilde{s}}+\kappa^{2} \phi(\kappa)
$$

where $\phi(\kappa)=\kappa^{1 / 3}$. Hence we can apply Matano's arguments [21] and conclude that the number of sign changes of $\kappa(\cdot, t)$ does not increase with time. Our main result in this section is an estimate for the number of such sign changes.

Theorem 9.1. Notation as above. If the total curvature $K=\int|\kappa| d \tilde{s}$ of the initial curve $\mathcal{C}_{0}$ is finite, then the number of (maximal) convex arcs at time $t_{*}>0$ does not exceed

$$
K \cdot \max \left(\frac{C L^{2}}{t_{*}^{3 / 2}}, \frac{2}{\pi}\right) .
$$

For comparison we remark that solutions of Euclidean curve shortening are real analytic, and hence always have a finite number of convex and concave arcs. Nonetheless no a priori estimate like (38) is known.

Throughout this section we will let $\mathcal{C}_{t}$ be a solution of affine curve shortening whose initial curve $\mathcal{C}_{0}$ is smooth and has only a finite number of inflection points. We will establish the estimate (38) for such curves with the constant $k$ independent of the solution $\mathcal{C}_{t}$. Approximation by analytic curves and passage to the limit then proves the general case.

9.1. Strong maximum principles for $\kappa$ and $\theta$. The equations for $\kappa$ and $\theta$ are degenerate so the strong maximum principle does not apply immediately.

Proposition 9.2. If $\kappa\left(p, t_{0}\right) \geq 0$ for $a \leq p \leq b$ and if $\kappa\left(a, t_{0}\right)>0, \kappa\left(b, t_{0}\right)>0$, then for some $\delta>0$ one has $\kappa(p, t)>0$ on $[a, b] \times\left(t_{0}, t_{0}+\delta\right)$.

Proposition 9.3. If $\theta\left(p, t_{0}\right) \geq \theta_{0}$ for $a \leq p \leq b$ and if $\theta\left(a, t_{0}\right)>\theta_{0}, \kappa\left(b, t_{0}\right)>0$, then for some $\delta>0$ one has $\theta(p, t)>\theta_{0}$ on $[a, b] \times\left(t_{0}, t_{0}+\delta\right)$.

Proof. Instead of considering the curves $\mathcal{C}_{t}$ directly, we let $\mathcal{C}_{t}^{\delta}$ be the solution of $\partial_{t} \mathcal{C}_{t}^{\delta}=\phi_{\delta}(\kappa) \mathcal{N}$, with $\phi_{\delta}$ as in (16), which at time $t=t_{0}$ coincides with $\mathcal{C}_{t_{0}}$. The velocity $v^{\delta}=\phi_{\delta}(\kappa)$ of $\mathcal{C}_{t}^{\delta}$ satisfies

$$
v_{t}^{\delta}=\phi_{\delta}^{\prime}(\kappa) v_{\tilde{s} \tilde{s}}^{\delta}+\kappa^{2} \phi_{\delta}^{\prime}(\kappa) v^{\delta} .
$$


Since $\kappa$ is bounded, uniformly in $\delta>0$, we have

$$
\phi_{\delta}^{\prime}(\kappa) \geq \eta>0, \quad 0 \leq \kappa^{2} \phi_{\delta}^{\prime}(\kappa) \leq M,
$$

for certain constants $0<\eta<M$. As $\delta \rightarrow 0$ the constant $\eta$ also decreases to 0 . We will assume below that $0<\eta<1$.

By continuity $v^{\delta}\left(a, t_{0}\right)>0$ implies that for some $\varepsilon>0$ one has $v^{\delta}(a, t)>0$ for $t_{0} \leq t \leq t_{0}+\varepsilon$. Similarly, $v^{\delta}\left(b, t_{0}\right)>0$ will hold for $t_{0} \leq t \leq t_{0}+\varepsilon$. The strong maximum principle forces $v^{\delta}(p, t)>0$ on the rectangle $\mathcal{R}=[\bar{a}, b] \times\left(t_{0}, t_{0}+\varepsilon\right)$. By letting $\delta$ drop to 0 we only get that $v(p, t) \geq 0$ on the rectangle $\mathcal{R}$, i.e., we get the weak maximum principle. To conclude $v>0$ for $\delta=0$, we must get an estimate for $v^{\delta}$ on $\mathcal{R}$. We do this with a subsolution for (39).

Let $\sigma^{\delta}(p, t)$ denote the arclength along $\mathcal{C}_{t}^{\delta}$ from $a$ to $p$. Then $\frac{\partial \sigma}{\partial \tilde{s}}=1$, and by the commutation relation $\left[\partial_{t}, \partial_{\tilde{s}}\right]=\kappa v \partial_{\tilde{s}}$ we find that

$$
\partial_{\tilde{s}}\left(\partial_{t} \sigma\right)=\partial_{t}(1)-\kappa v \partial_{\tilde{s}} \sigma=-\kappa v,
$$

which is uniformly bounded. Together with $\sigma^{\delta}(a, t) \equiv 0$ this implies that $\partial_{t} \sigma^{\delta}$ and hence $\sigma^{\delta}$ are bounded (uniformly in $\delta$ ).

Consider

$$
w^{\delta}(p, t)=\alpha \Gamma\left(\sigma^{\delta}(p, t)+\gamma\left(t-t_{0}\right)+1, \eta\left(t-t_{0}\right)\right)
$$

where $\alpha, \gamma$ are constants and

$$
\Gamma(x, \tau)=\frac{1}{\sqrt{ } \tau} \exp \left\{\frac{-x^{2}}{4 \tau}\right\}
$$

is the fundamental solution of the heat equation $\Gamma_{\tau}=\Gamma_{x x}$. If $x>0$, then $\Gamma(x, \tau)$ is decreasing $\left(\Gamma_{x}<0\right)$, and if $x>2 \sqrt{ } \tau$, then $\Gamma(x, \tau)$ is convex $\left(\Gamma_{x x}>0\right)$ as a function of $x$.

On the rectangle $\mathcal{R}$ we have $\sigma(p, t) \geq 0$ and $0 \leq t-t_{0} \leq \varepsilon$ and hence $x=$ $\sigma(p, t)-\gamma\left(t-t_{0}\right)+1$ and $\tau=\eta\left(t-t_{0}\right)$ satisfy

$$
x^{2} \geq(1-\gamma \varepsilon)^{2}>2 \varepsilon>2 \eta \varepsilon \geq 2 \tau
$$

provided $\varepsilon$ is chosen small enough (remember that $\eta<1$ ).

How small $\varepsilon$ should be chosen depends on $\gamma$. If we now choose $\gamma>-\inf \sigma_{t}^{\delta}$, then we get

$$
\begin{aligned}
w_{t}^{\delta} & =\eta \alpha \Gamma_{\tau}+\alpha\left(\gamma+\sigma_{t}^{\delta}\right) \Gamma_{x} \\
& =\eta \alpha \Gamma_{x x}+\alpha\left(\gamma+\sigma_{t}^{\delta}\right) \Gamma_{x} \\
& =\eta w_{\tilde{s} \tilde{s}}^{\delta}+\alpha\left(\gamma+\sigma_{t}^{\delta}\right) \Gamma_{x} \\
& \leq \phi_{\delta}^{\prime}(\kappa) w_{\tilde{s} \tilde{s}}^{\delta} \\
& \leq \phi_{\delta}^{\prime}(\kappa) w_{\tilde{s} \tilde{s}}^{\delta}+\kappa^{2} \phi_{\delta}^{\prime}(\kappa) w^{\delta} .
\end{aligned}
$$

Hence $w^{\delta}(p, t)$ is a subsolution on $\mathcal{R}$ for all small $\delta>0$. At $t=t_{0}$ we have $w^{\delta}(p, t) \equiv 0$. If we choose $\alpha>0$ sufficiently small, then we will have $w^{\delta} \leq v^{\delta}$ on the vertical sides of $\mathcal{R}$ for all $\delta>0$. The maximum principle then implies $\overline{v^{\delta}} \geq w^{\delta}$ on $\mathcal{R}$. In the limit $\delta \rightarrow 0$ we find that $v(p, t) \geq w^{0}(p, t)>0$ on $\mathcal{R}$. This completes the proof of Proposition 9.2.

The proof of Proposition 9.3 is very similar. One notes that the angle $\theta^{\delta}(p, t)$ of the approximating evolutions evolve according to

$$
\frac{\partial \theta}{\partial t}=\frac{\partial v}{\partial \tilde{s}}=\phi_{\delta}^{\prime}(\kappa) \frac{\partial^{2} \theta}{\partial \tilde{s}^{2}} .
$$


Thus for any constant $\theta_{0}$ the function $w=\theta^{\delta}-\theta_{0}$ evolves according to $w_{t}=$ $\phi_{\delta}^{\prime}(\kappa) w_{\tilde{s} \tilde{s}}$. One can then use the same subsolution (40) to prove $\theta>\theta_{0}$ on $\mathcal{R}$.

9.2. History of a convex arc. In what follows below, we will just consider convex arcs. The treatment for concave arcs is of course identical.

Let $\beta=\left\{X\left(p, t_{*}\right): p_{-} \leq p \leq p_{+}\right\}$be a given convex arc.

Since $\kappa\left(\cdot, t_{*}\right)$ only has a finite number of sign changes, there is an interval $\left(p_{-}-\varepsilon, p_{-}\right)$on which $\kappa\left(p, t_{*}\right)<0$. Let $\mathcal{O}_{-}$be the connected component of the set $\left\{(p, t) \in \mathbf{R} \times\left[0, t_{*}\right]: \kappa(p, t)<0\right\}$ which contains the segment $\left(p_{-}-\varepsilon, p_{-}\right) \times\left\{t_{*}\right\}$. Similarly we define $\mathcal{O}_{+}$to be the component of $\{\kappa<0\}$ which meets the segment $\left(p_{+}, p_{+}+\varepsilon\right) \times\left\{t_{*}\right\}$.

Since the curvature satisfies the weak maximum principle (see Lemma 7.1), Matano's arguments in [21] can be applied to prove:

Lemma 9.4 (Matano). For any $\left(p_{0}, t_{0}\right) \in \mathbf{R} \times\left(0, t_{*}\right)$ with $\kappa\left(p_{0}, t_{0}\right) \neq 0$ there is a continuous $P:\left[0, t_{0}\right] \rightarrow \mathbf{R}$ with $P\left(t_{0}\right)=p_{0}$ and $\kappa(P(t), t) \neq 0$ for all $0 \leq t \leq t_{0}$.

Hence there exists some $p$ with $(p, t) \in \mathcal{O}_{-}$for every $t \in\left[0, t_{*}\right]$, and we may define

$$
\begin{aligned}
& p_{-}(t)=\sup \left\{p:(p, t) \in \mathcal{O}_{-}\right\}, \\
& p_{+}(t)=\inf \left\{p:(p, t) \in \mathcal{O}_{+}\right\} .
\end{aligned}
$$

For $0 \leq t \leq t_{*}$, we let $\beta_{t}$ be the image under $X(\cdot, t)$ of $\left[p_{-}(t), p_{+}(t)\right]$. By definition, $p_{-}\left(t_{*}\right)=p_{-}$and $p_{+}\left(t_{*}\right)=p_{+}$, so $\beta_{t_{*}}=\beta$. We call $\left\{\beta_{t}: 0 \leq t \leq t_{*}\right\}$ the history of the $\operatorname{arc} \beta_{t_{*}}$.

Proposition 9.5. Disjoint arcs $\beta_{1}, \beta_{2} \subset \mathcal{C}_{t}$ have disjoint histories.

Proof. Let

$$
\beta_{1} \stackrel{\text { def }}{=} X\left(\left[p_{1,-}, p_{1,+}\right], t_{*}\right), \quad \beta_{2} \stackrel{\text { def }}{=} X\left(\left[p_{2,-}, p_{2,+}\right], t_{*}\right),
$$

and assume that $p_{1,-}<p_{1,+}<p_{2,-}<p_{2,+}$. Then by the maximality of $\beta_{i}, i=1,2$, there is a $q \in\left(p_{1,+}, p_{2,-}\right)$ with $\kappa\left(q, t_{*}\right)<0$. By Matano's Lemma 9.4, there must be a continuous $q:\left[0, t_{*}\right] \rightarrow \mathbf{R}$ with $\kappa(q(t), t)<0$ and $q\left(t_{*}\right)=q$. But then $p_{1,+}(t)<q(t)<p_{2,-}(t)$ for $0 \leq t \leq t_{*}$.

\subsection{Range of the tangent angle. Set}

$$
\Sigma_{t}:=\left\{\theta(p, t): p_{-}(t) \leq p \leq p_{+}(t)\right\},
$$

where $\theta(\cdot, t)$ denotes the standard angle parameter on $\beta_{t}$.

Lemma 9.6. If $0 \leq t_{1}<t_{2} \leq t_{*}$, then $\Sigma_{t_{1}} \supset \Sigma_{t_{2}}$.

In the proof of this proposition it will be convenient to define

$$
q_{-}(t)=\inf \left\{p>p_{-}(t): \kappa(p, t)>0\right\}, \quad q_{+}(t)=\sup \left\{p<p_{+}(t): \kappa(p, t)>0\right\} .
$$

Proposition 9.7. $\kappa(p, t) \equiv 0$ for $p_{-}(t) \leq p \leq q_{-}(t)$.

Proof. Since $\kappa(\cdot, t)$ only has finitely many sign changes, $\kappa(p, t)$ must be positive for all $p$ in some small interval $\left(q_{-}(t), q_{-}(t)+\varepsilon\right)$. In the interval $\left[p_{-}(t), q_{-}(t)\right]$ one must have $\kappa(p, t) \leq 0$, by virtue of the definition of $q_{-}(t)$. To see that $\kappa$ actually vanishes on this interval we assume the contrary, i.e., that $\kappa\left(p_{0}, t_{0}\right)<0$ for some $t_{0}$ and $p_{0} \in\left(p_{-}\left(t_{0}\right), q_{-}\left(t_{0}\right)\right)$. For some $\varepsilon>0$ one has $\kappa\left(p, t_{0}\right)<0$ for $p_{-}\left(t_{0}\right)-\varepsilon<p<p_{-}\left(t_{0}\right)$. By Proposition 9.2 we then would have $\kappa<0$ on 
some rectangle $\mathcal{R}=\left(p_{-}\left(t_{0}\right)-\varepsilon, p_{0}\right) \times\left(t_{0}, t_{0}+\varepsilon\right)$. One could then connect $\left(p_{0}, t_{0}\right)$ to $\left(p_{-}\left(t_{0}\right)-\varepsilon, t_{0}\right)$ within $\{\kappa<0\}$, so that $\left(p_{0}, t_{0}\right) \in \mathcal{O}_{-}$. This contradicts the definition of $p_{-}\left(t_{0}\right)$.

Proposition 9.8. $p_{-}(t)$ is a lower semicontinuous function; $p_{+}(t)$ is upper semicontinuous. Furthermore, we have

$$
\begin{gathered}
\limsup _{t \rightarrow t_{0}} p_{-}(t) \leq q_{-}\left(t_{0}\right), \\
\liminf _{t \rightarrow t_{0}} p_{+}(t) \geq q_{+}\left(t_{0}\right) .
\end{gathered}
$$

Proof. Lower semicontinuity of $p_{-}(t)$ follows from openness of $\mathcal{O}_{-}$: given $t_{0}$ and small $\varepsilon>0$, one has $\left(p_{-}\left(t_{0}\right), t_{0}\right) \in \mathcal{O}_{-}$and hence for small $\delta>0$ one has $\left(p_{-}\left(t_{0}\right), t\right) \in \mathcal{O}_{-}$for all $t \in\left(t_{0}-\delta, t_{0}+\delta\right)$. Hence $p_{-}(t)>p_{-}\left(t_{0}\right)-\delta$ for $\left|t-t_{0}\right|<\delta$. Openness of $\mathcal{O}_{+}$similarly implies upper semicontinuity of $p_{+}(t)$.

For small $\varepsilon>0$ one will have $\kappa\left(q_{-}\left(t_{0}\right)+\varepsilon, t_{0}\right)>0$. By continuity of $\kappa$ one also has $\kappa\left(q_{-}\left(t_{0}\right)+\varepsilon, t\right)>0$ for $\left|t-t_{0}\right|<\delta$, if $\delta$ is small enough. Matano's lemma provides a continuous $P:\left[0, t_{0}-\delta\right] \rightarrow \mathbf{R}$ with $\kappa(P(t), t)>0$ and $P\left(t_{0}-\delta\right)=q_{-}\left(t_{0}\right)+\varepsilon$. Let $\Gamma$ be the segment $\left\{q_{-}\left(t_{0}\right)+\varepsilon\right\} \times\left[t_{0}-\delta, t_{0}+\delta\right]$.

Suppose that $p_{-}\left(t_{1}\right)>q_{-}\left(t_{0}\right)+\varepsilon$ for some $t_{1} \in\left(t_{0}-\delta, t_{0}+\delta\right)$. Then $\left(p_{1}, t_{1}\right) \in$ $\mathcal{O}_{-}$for some $p_{1} \in\left(q_{-}\left(t_{0}\right)+\varepsilon, p_{-}\left(t_{1}\right)\right)$, which means one can connect $\left(p_{1}, t_{1}\right)$ to $\left(p_{-}\left(t_{*}\right)-\varepsilon, t_{*}\right)$ with some graph $p=P_{*}^{(1)}(t), t_{1} \leq t \leq t_{*}$, in $\mathcal{O}_{-}$. By Matano's lemma one can also find a continuous function $p=P_{*}^{(2)}(t), 0 \leq t \leq t_{1}$, whose graph lies in $\mathcal{O}_{-}$and goes through $\left(p_{1}, t_{1}\right)$.

Let $P_{*}:\left[0, t_{*}\right] \rightarrow \mathbf{R}$ be the function obtained by combining $P_{*}^{(1)}$ and $P_{*}^{(2)}$. Then the graph of $P_{*}$ lies in $\mathcal{O}_{-}$, and since $\kappa>0$ on the segment $\Gamma$, the graph of $P_{*}$ must be disjoint from $\Gamma$, and so one has $P_{*}(t)>q_{-}\left(t_{0}\right)+\varepsilon$ for $\left|t-t_{0}\right| \leq \delta$. In particular $P\left(t_{0}\right)>p_{-}\left(t_{0}\right)$, which contradicts the definition of $p_{-}\left(t_{0}\right)$.

To complete the proof of Lemma 9.6 we now show that

$$
\theta_{-}(t) \stackrel{\text { def }}{=} \theta\left(p_{-}(t), t\right)
$$

is a non-increasing function of $t$. To begin we note that

$$
\theta_{-}(t)=\theta(p, t) \text { for all } p \in\left[p_{-}(t), q_{-}(t)\right]
$$

since $\kappa(p, t) \equiv 0$ on that interval. It then follows from continuity of $\theta: \mathbf{R} \times\left[0, t_{*}\right] \rightarrow$ $\mathbf{R}$ and Proposition 9.8 that $\theta_{-}(t)$ is a continuous function.

At any given $t_{0}$ there will be an $\varepsilon>0$ such that $\kappa\left(\cdot, t_{0}\right)<0$ on $\left[p_{-}\left(t_{0}\right)-\varepsilon, p_{-}\left(t_{0}\right)\right)$ and $\kappa\left(\cdot, t_{0}\right)>0$ on $\left(q_{-}\left(t_{0}\right), q_{-}\left(t_{0}\right)+\varepsilon\right]$. Thus $\theta\left(p, t_{0}\right) \geq \theta_{-}\left(t_{0}\right)$ on the closed interval $\left[p_{-}\left(t_{0}\right)-\varepsilon, q_{-}\left(t_{0}\right)+\varepsilon\right]$, with strict inequality at the endpoints of this interval. By Proposition 9.3 we then get $\theta(p, t)>\theta_{-}\left(t_{0}\right)$ on some rectangle $\left[p_{-}\left(t_{0}\right)-\varepsilon\right.$, $\left.q_{-}\left(t_{0}\right)+\varepsilon\right] \times\left(t_{0}, t_{0}+\delta\right)$. Since $p_{-}(t)$ lies between $p_{-}\left(t_{0}\right)-\varepsilon$ and $q_{-}\left(t_{0}\right)+\varepsilon$ for $t$ close to $t_{0}$, we see that $\theta_{-}(t)>\theta_{-}\left(t_{0}\right)$ for $t_{0}<t<t_{0}+\delta$. Hence $\theta_{-}(t)$ is an increasing function, as claimed.

The same arguments show that $\theta_{+}(t) \stackrel{\text { def }}{=} \theta_{+}\left(p_{+}(t), t\right)$ is a decreasing function. Lemma 9.6 now follows from $\Sigma(t)=\left[\theta_{-}(t), \theta_{+}(t)\right]$.

9.4. Total curvature of the history. Now define

$$
\Delta \theta(t):=\sup \left\{\left|\theta\left(p_{1}, t\right)-\theta\left(p_{2}, t\right)\right|: p_{-}(t) \leq p_{1}, p_{2} \leq p_{+}(t)\right\} .
$$


Set

the length of the initial curve $\mathcal{C}_{0}$.

$$
L:=L\left(\mathcal{C}_{0}\right)
$$

We will now prove the following:

Proposition 9.9. Notation as above. Let $\beta_{t_{*}} \subset \mathcal{C}_{t_{*}}$ at time $t_{*}>0$ be a (maximal) convex arc. Then

$$
\Delta \theta(0) \geq \min \left(\frac{t_{*}^{\frac{3}{2}}}{C L^{2}}, \frac{\pi}{2}\right),
$$

for some constant $C$.

Since convex and concave arcs alternate, we get the same estimate for concave arcs.

9.5. Proof of Proposition 9.9 and Theorem 9.1. Assume that $\Delta \theta(0)<\frac{\pi}{2}$, and let

$$
\Sigma_{0} \subset\left[-\frac{\Delta \theta(0)}{2}, \frac{\Delta \theta(0)}{2}\right] .
$$

On any convex part of $\beta_{t}$, we can use the angle parameter $\theta$, and then the normal velocity as a function of $\theta, t$ satisfies

$$
\frac{\partial v}{\partial t}=\frac{1}{3} v^{4}\left(v_{\theta \theta}+v\right)
$$

A computation shows that

$$
\bar{v}(\theta, t)=A(t) \cos \left(\frac{\pi \theta}{2 \Delta \theta(0)}\right)
$$

will be a supersolution of (42), if we choose

$$
A(t)=\left\{A(0)^{-4}+\frac{c t}{(\Delta \theta)^{2}}\right\}^{-\frac{1}{4}},
$$

for $c$ a suitably small constant.

Now by the maximum principle we get the following inequalities for $v$ : First choosing $A(0)=\infty$, we see that

$$
|v(\theta, t)| \leq \frac{c(\Delta \theta(0))^{1 / 2}}{t^{1 / 4}} .
$$

Second, if one takes $A(0)=\left(\frac{1}{15}\right)^{1 / 4} \sup _{\beta_{0}}|v|$, then we find

$$
|v(\theta, t)| \leq\left(15+\frac{c t}{(\Delta \theta)^{2}} \sup _{\beta_{0}} v^{4}\right)^{-1 / 4} \sup _{\beta_{0}}|v| .
$$

A word of explanation is in order about our use of the maximum principle here since the equation for $v(42)$ is degenerate at $v=0$, and the domain of $\theta$ is not fixed. In fact, for one value of $\theta, v(\theta, t)$ may have several values if there are several convex/concave arcs in $\beta_{t}$ with tangent in the direction of $\theta$. Nevertheless, it is easy to justify the use of the maximum principle in this context. Indeed, at any $t \in$ $\left(0, t_{*}\right), \beta_{t}$ is the union of a finite number of convex/concave arcs. On each of these arcs, the angle $\theta$ is a single-valued coordinate which takes values in some interval contained in $\left[-\frac{1}{2} \Delta \theta(t), \frac{1}{2} \Delta \theta(t)\right]$. This interval changes in time, but at its endpoints $v$ vanishes, while our supersolution $\bar{v}$ is bounded from below on $\left[-\frac{1}{2} \Delta \theta(t), \frac{1}{2} \Delta \theta(t)\right]$. 
Thus the maximum principle does indeed apply and gives $v \leq \bar{v}$ on convex arcs, and $-v \leq \bar{v}$ on concave arcs. Hence we have

$$
|v(p, t)| \leq \bar{v}(\theta(p, t), t) \text {, on } \beta_{t} .
$$

Next the estimate (46) implies that at

$$
t=T_{1 / 2}\left(\sup _{\beta_{0}}|v|, \Delta \theta\right):=\frac{(\Delta \theta)^{2}}{c\left(\sup _{\beta_{0}}|v|\right)^{4}}, \quad \Delta \theta=\Delta \theta(0),
$$

one has

$$
\sup _{\beta_{T_{1 / 2}}}|v| \leq \frac{1}{2} \sup _{\beta_{0}}|v|
$$

By the same arguments, we get

$$
\sup _{\beta_{t+T_{1 / 2}}}|v| \leq \frac{1}{2} \sup _{\beta_{t}}|v|
$$

for $T_{1 / 2}=T_{1 / 2}\left(\sup _{\beta_{t}}|v|, \Delta \theta\right)$ as given in (47), with

$$
\Delta \theta=\Delta \theta(t)=\sup \left\{\left|\theta\left(p_{1}, t\right)-\theta\left(p_{2}, t\right)\right|: p_{-}(t) \leq p_{1}, p_{2} \leq p_{+}(t)\right\} .
$$

We now inductively define a sequence $t_{0}<t_{1}<t_{2}<\cdots$, with

$$
\sup _{\beta_{t_{j+1}}}|v| \leq \frac{1}{2} \sup _{\beta_{t_{j}}}|v|
$$

by putting

$$
t_{j+1}-t_{j}=T_{1 / 2}\left(\sup _{\beta_{t_{j}}}|v|, \Delta \theta\left(t_{j}\right)\right) .
$$

Setting $L=L\left(\mathcal{C}_{0}\right)$, we may (crudely) estimate $\Delta \theta\left(t_{j}\right)$ by

$$
\Delta \theta\left(t_{j}\right) \leq \int_{\beta\left(t_{j}\right)}|\kappa| d \tilde{s} \leq\left(\sup _{\beta_{t_{j}}}|v|\right)^{3} L,
$$

so that

$$
T_{1 / 2}\left(\sup _{\beta_{t_{j}}}|v|, \Delta \theta\left(t_{j}\right)\right) \leq \frac{L^{2}}{c}\left(\sup _{\beta_{t_{j}}}|v|\right)^{2} .
$$

From

$$
\sup _{\beta_{t_{j}}}|v| \leq 2^{-j} \sup _{\beta_{0}}|v|
$$

we find

$$
t_{j+1}-t_{j} \leq \frac{L^{2}}{c} 2^{-2 j}\left(\sup _{\beta_{t_{0}}}|v|\right)^{2}
$$

so that

$$
t_{\infty}:=\lim _{j \rightarrow \infty} t_{j} \leq t_{0}+C L^{2}\left(\sup _{\beta_{t_{0}}}|v|\right)^{2},
$$

where $C$ is a constant. We can estimate $\sup _{\beta_{t_{0}}}|v|$ using (45), which gives

$$
t_{\infty} \leq t_{0}+\frac{C L^{2} \Delta \theta(0)}{t_{0}^{1 / 2}} .
$$

The best estimate is obtained for

$$
t_{0}=\left(2 C L^{2} \Delta \theta(0)\right)^{2 / 3},
$$


which gives

$$
t_{\infty} \leq\left(C L^{2} \Delta \theta(0)\right)^{2 / 3} .
$$

Since our maximal arc $\beta_{t_{*}}$ is not flat, we must have $t_{\infty} \geq t_{*}$, that is,

$$
\Delta \theta(0) \geq \frac{t_{*}^{2 / 3}}{C L^{2}},
$$

provided $\Delta \theta(0) \leq \pi / 2$ (a condition which we assumed above). This proves Proposition 9.9 .

For the proof of Theorem 9.1, we note that since disjoint maximal convex arcs $\beta_{t_{*}} \subset \mathcal{C}_{t_{*}}$ have disjoint histories, there cannot be more than

$$
\max \left(\frac{K C L^{2}}{t_{*}^{3 / 2}}, \frac{2 K}{\pi}\right)
$$

convex $\operatorname{arcs}$ in $\mathcal{C}_{t_{*}}$, where $K$ denotes the total curvature of $\mathcal{C}_{0}$.

\subsection{Arcs with large curvature.}

Lemma 9.10. For any solution $\left\{\mathcal{C}_{t} \mid 0<t<T\right\}$ of Affine Curve Shortening (15) there is a constant $K$ such that the total curvature $\left|\int \kappa d \tilde{s}\right|$ of any convex or concave arc with $\sup |\kappa|>K$ exceeds $\pi$.

This lemma was proved by Grayson [16, Lemma 3.5] for Euclidean Curve Shortening. The two ingredients in his proof are (1) finiteness of the number of convex and concave arcs, and (2) the steady (super)solution $\kappa(\theta)=A \sin (\theta-\alpha)$ for the curvature equation. We have just shown that solutions of Affine Curve Shortening also break up into a finite number of convex and concave arcs, and instead of using the curvature equation we can use equation (42) for the velocity: $v(\theta)=A \sin (\theta-\alpha)$ is also a supersolution for this equation. With these remarks we may simply repeat Grayson's arguments to prove Lemma 9.10.

\section{INTERSECTION OF SOLUTIONS}

Let $\mathcal{C}_{1}, \mathcal{C}_{2} \subset \mathbf{R}^{2}$ be embedded $C^{1}$ curves. Suppose that $\mathcal{C}_{1}$ and $\mathcal{C}_{2}$ intersect tranversally, i.e., the unit tangents at a given point of intersection are independent. In this case, we define $\#\left(\mathcal{C}_{1} \cap \mathcal{C}_{2}\right)$, the number of crossings of $\mathcal{C}_{1}$ and $\mathcal{C}_{2}$, to be the number of intersection points of $\mathcal{C}_{1}$ and $\mathcal{C}_{2}$.

If $\mathcal{C}_{1}$ and $\mathcal{C}_{2}$ do not intersect tranversally, we choose a tubular neighborhood $\mathbf{N}$ of $\mathcal{C}_{1}$, and then decompose $\mathcal{C}_{2} \cap \mathbf{N}$ into pieces which are graphs in $\mathbf{N}$. If we identify $\mathcal{C}_{1}$ with the zero section of $\mathbf{N}$, we can count the number of sign changes of each of those pieces. The resulting sum is by definition $\#\left(\mathcal{C}_{1} \cap \mathcal{C}_{2}\right)$, the number of crossings of $\mathcal{C}_{1}$ and $\mathcal{C}_{2}$. (This definition is the same as in [5].)

Theorem 10.1 (Weak Sturmian Theorem). Let $\mathcal{C}_{1}(\cdot, t), \mathcal{C}_{2}(\cdot, t)$ evolve by $v=\kappa^{1 / 3}$. Then

$$
\#\left(\mathcal{C}_{1}(\cdot, t) \cap \mathcal{C}_{2}(\cdot, t)\right) \leq \#\left(\mathcal{C}_{1}(\cdot, 0) \cap \mathcal{C}_{2}(\cdot, 0)\right) .
$$

Proof. This weaker version of the results in $[5,7]$ may be proved using Matano's method [21] which only uses the weak maximum principle (see also Lemma 9.4).

It is important to note that the weak Sturmian theorem does not claim that $\#\left(\mathcal{C}_{1}(\cdot, t) \cap \mathcal{C}_{2}(\cdot, t)\right)$ is always finite, or that non-transverse intersections of $\mathcal{C}_{1}(\cdot, t)$ and $\mathcal{C}_{2}(\cdot, t)$ cause $\#\left(\mathcal{C}_{1}(\cdot, t) \cap \mathcal{C}_{2}(\cdot, t)\right)$ to decrease (see $\left.[5,7]\right)$. In this regard, we have the following illuminating examples: 


\subsection{Some special solutions of Affine Curve Shortening. Let}

$$
u(x, t)=(T-t)^{\alpha} \Phi(x) .
$$

Then

$$
u_{t}-\left(u_{x x}\right)^{1 / 3}=\alpha(T-t)^{\alpha-1} \Phi(x)-(T-t)^{\alpha / 3}\left(\Phi^{\prime \prime}(x)\right)^{1 / 3} .
$$

Thus $u$ will be a solution of the local affine heat equation (33) if $\alpha=3 / 2$ and

$$
\Phi^{\prime \prime}(x)=\left(\frac{3}{2} \Phi(x)\right)^{3} .
$$

The latter ODE has oscillatory solutions which can be written in terms of elliptic functions. These special solutions intersect the $x$-axis only finitely often on any bounded interval for $t<T$, but coalesce with the $x$-axis at $t=T$. Consequently, the strong maximum principle and backward uniqueness fail for the affine heat equation (33).

We now look for examples of solutions of (33) of the form

$$
u(x, t)=\Phi\left(x t^{-\alpha}\right) .
$$

We compute that with $\xi \stackrel{\text { def }}{=} x t^{-\alpha}$,

$$
\begin{aligned}
u_{t}-\left(u_{x x}\right)^{1 / 3} & =\frac{-\alpha x}{t^{\alpha+1}} \Phi^{\prime}(\xi)-t^{-2 \alpha / 3}\left(\Phi^{\prime \prime}(\xi)\right)^{1 / 3} \\
& =\frac{-\alpha}{t} \xi \Phi^{\prime}(\xi)-t^{-2 \alpha / 3}\left(\Phi^{\prime \prime}(\xi)\right)^{1 / 3} .
\end{aligned}
$$

Take $\alpha=3 / 2$, and solve the resulting equation

$$
\left(\Phi^{\prime \prime}(\xi)\right)^{1 / 3}+\frac{3}{2} \xi \Phi^{\prime}(\xi)=0 .
$$

It is then easy to see that

$$
\begin{aligned}
\Phi^{\prime \prime}(\xi) & =\frac{-27}{8} \xi^{3} \Phi^{\prime}(\xi)^{3}, \\
\left(\Phi^{\prime}(\xi)^{-2}\right)^{\prime} & =\frac{27}{4} \xi^{3}=\left(\frac{27}{16} \xi^{4}\right)^{\prime}, \\
\Phi^{\prime}(\xi) & =-\frac{4}{3 \sqrt{3}} \frac{1}{\sqrt{A+\xi^{4}}} \text { for some constant } A,
\end{aligned}
$$

from which we derive the step function solution

$$
\Phi(\xi)=\frac{4}{3 \sqrt{3}} \int_{\xi}^{\infty} \frac{1}{\sqrt{A+s^{4}}} d s .
$$

Thus we get a solution of the affine heat equation (33) of the form

$$
u_{A}(x, t)=\Phi_{A}\left(\frac{x}{t^{3 / 2}}\right),
$$

where $\Phi_{A}(\xi)$ is given by (51). This solution is the affine analogue of the error function solution of the classical linear heat equation $u_{t}=u_{x x}$.

The initial value is

$$
u_{A}(x, 0)=\left\{\begin{array}{cc}
0 & (x>0) \\
\Phi_{A}(-\infty) & (x<0)
\end{array}\right.
$$

where

$$
\Phi_{A}(-\infty)=\frac{4}{3 \sqrt{3}} \int_{-\infty}^{\infty} \frac{1}{\sqrt{A+s^{4}}} d s=\frac{4 A^{-1 / 4}}{3 \sqrt{3}} \int_{-\infty}^{\infty} \frac{1}{\sqrt{1+s^{4}}} d s
$$


A study of the inflection point $x=0$ of $u_{A}(x, t)$ for given $t>0$, shows that it indeed has fifth order contact with its tangent, as predicted by the theory.

\section{Evolving Foliated RECTANGLes}

In this section, we will use the technique of foliated rectangles in order to prevent singularity formation in the equation (15). We begin with:

Definition. A foliated rectangle $\mathcal{F}$ is a family of $C^{1}$ functions

$$
\left\{u^{a} \in C^{1}\left(\left[x_{0}, x_{1}\right]\right): a_{0} \leq a \leq a_{1}\right\},
$$

for which

(1) $u^{a}(x), u_{x}^{a}(x)$ are continuous in $(a, x)$.

(2) If $a<a^{\prime}$, then $u^{a}(x)<u^{a^{\prime}}(x)$ for $x_{0} \leq x \leq x_{1}$.

Definition. An evolving foliated rectangle

$$
\left\{\mathcal{F}_{t}: 0<t<T\right\}
$$

is a family of foliated rectangles $\mathcal{F}_{t}$ given by $u^{a}(x, t)$, where each $u^{a}(x, t)$ is a classical solution of the affine heat equation $u_{t}=\left(u_{x x}\right)^{1 / 3}$ with $u^{a}\left(x_{0}, t\right)$ and $u^{a}\left(x_{1}, t\right)$ constant.

Lemma 11.1. Every "initial" foliated rectangle $\mathcal{F}_{0}$ defines a unique evolving foliated rectangle $\left\{\mathcal{F}_{t}: 0<t<\infty\right\}$.

Proof. Let $\mathcal{F}_{0}$ be defined by $\left\{u^{a}: a_{0} \leq a \leq a_{1}\right\}, u^{a} \in C^{1}\left(\left[x_{0}, x_{1}\right]\right)$. Without loss of generality, we may assume that $x_{0}=0, x_{1}=1, a_{0}=0, a_{1}=1$. We may extend the $u^{a}$ by reflection, so that $u_{x}^{a}$ is an odd and periodic function of period 2 . We first prove Lemma 11.1 under the assumption that the $u^{a}$ are $C^{2}$. In this case, the same arguments leading to the short-term existence theorem (Theorem 5.2) imply the existence on a short-term time interval of a classical solution $u^{a}: \mathbf{R} \times(0, T) \rightarrow \mathbf{R}$ with the given initial data.

Now intersection with steep straight lines shows that

$$
\sup _{\mathbf{R}}\left|\frac{\partial u^{a}}{\partial x}\right|
$$

does not increase. From Theorem 8.1 we get that

$$
\left|u_{x x}^{a}\right| \leq \frac{C}{t^{3 / 4}}
$$

for some constant $C$ and $0<t<\infty$. Hence the curvature of the $u^{a}$ cannot blow up in finite time, so the $u^{a}$ are defined for all $t \geq 0$. Moreover, the decay estimate (52) suggests that

$$
u^{a}(x, t) \rightarrow u^{a}(0)(1-x)+u^{a}(1) x, \text { as } t \rightarrow \infty .
$$

In fact, by comparing with the solution of Example 1 (showing the strong maximum does not hold for the affine heat equation), from equation (50), one can show that the graphs of the $u^{a}$ become straight lines in finite time. (This follows from the fact that $u(x, t)=\lambda(T-t)^{3 / 2} \Phi(\lambda x)+\mu x+\nu$ is a solution of (33), if $\Phi$ satisfies (50).)

To prove that the $\left\{u^{a}(\cdot, t): 0 \leq a \leq 1\right\}$ form a foliated rectangle, we observe that for $a, a^{\prime} \in[0,1]$, the maximum principle implies

$$
\sup _{x}\left|u^{a}(x, t)-u^{a^{\prime}}(x, t)\right| \leq \sup _{x}\left|u^{a}(x, 0)-u^{a^{\prime}}(x, 0)\right|,
$$


and if $a>a^{\prime}$, then

$$
\inf _{x}\left(u^{a}(x, t)-u^{a^{\prime}}(x, t)\right) \geq \inf _{x}\left(u^{a}(x, 0)-u^{a^{\prime}}(x, 0)\right) .
$$

Inequality (53) implies that for a sequence $a_{n} \rightarrow a$, the $u^{a_{n}}(\cdot, t)$ converge uniformly to $u^{a}(\cdot, t)$, and because of the derivative bounds given above also in $C^{2}([0,1])$. Then inequality (54) implies that $u^{a}>u^{a^{\prime}}$ for $a>a^{\prime}$. Thus the proof of the lemma is complete if the initial $\mathcal{F}_{0}$ is $C^{2}$.

For $C^{1}$ initial foliated rectangles, we can approximate by smooth foliated rectangles. The derivative bounds only depend on

$$
\sup _{a, x}\left|u_{x}^{a}\right|,
$$

so we can extract convergent subsequences. Uniqueness of the resulting $\mathcal{F}_{t}$ follows from (53).

We now prove a result which prevents the formation of singularities of the flow (15) using the technique of evolving foliated rectangles.

Theorem 11.2. Let $\left\{\mathcal{F}_{t}: 0<t<T\right\}$ be an evolving foliated rectangle. Let $\left\{\mathcal{C}_{t}\right.$ : $0<t<T\}$ be a classical solution of (15) which never hits the edges of $\mathcal{F}_{t}$, and such that $\mathcal{C}_{t}$ is transverse to each leaf of $\mathcal{F}_{0}$ and intersects it once. Then $\mathcal{C}_{t}$ does not form a singularity in the interior of $\mathcal{F}_{T}$.

Proof. The main ideas are based on [5]; see the proof of Theorem 7.1. Let $\mathcal{F}_{t}$ be given by

$$
y=u^{a}(x, t), \quad 0 \leq a \leq 1 .
$$

From the affine invariance of (33), it is easily verified that the functions

$$
u^{a, \alpha, \beta}(x, t)=u^{a}(x, t)+\alpha x+\beta
$$

are solutions of (33). By compactness, there is an $\varepsilon_{0}>0$ such that $\mathcal{C}_{0}$ is transverse to the graph of any $u^{a, \alpha, \beta}$ with $0 \leq a \leq 1,|\alpha|,|\beta| \leq \varepsilon_{0}$, and $\mathcal{C}_{t}$ is disjoint from the endpoints of the $u^{a, \alpha, \beta}(0 \leq t<T)$. (Note that since $\mathcal{C}_{t}$ is a closed curve, it will always intersect each leaf of $\mathcal{F}_{t}$.)

Let $P=\left(x_{0}, u^{a}\left(x_{0}, t_{0}\right)\right)$, where $0 \leq t_{0}<T$. Then if $C_{t_{0}}$ passes through $P$, it will have at most one crossing with the graph of

$$
y=u^{a}(x, t)+\alpha\left(x-x_{0}\right)=u^{a, \alpha,-\alpha x_{0}}(x, t),
$$

for any $\alpha$ with $|\alpha|,\left|\alpha x_{0}\right| \leq \varepsilon_{o}$. This implies that the $\mathcal{C}_{t}$ are uniformly locally Lipschitz in $\mathcal{F}_{t}$. Hence $\mathcal{C}_{t}$ cannot have a singularity in $\mathcal{F}_{T}$.

Remark. The point of the above proof is that the $u^{a, \alpha, \beta}$ define a cone-field on $\mathcal{F}_{t}$ to which $\mathcal{C}_{t}$ is transverse. This forces $\mathcal{C}_{t}$ to be uniformly locally Lipschitz.

\section{THE $2 \pi$-THEOREM}

In this section, we prove one of the key results needed to guarantee the convergence of a given curve under the affine flow (15) to an elliptical point.

We first note that as in the curve shortening (Euclidean) flow [16], for the affine flow (15), the total curvature of $\mathcal{C}_{t}$ does not increase (see also [31]). 
Let $\psi$ be a convex $C^{2}$ function. Then

$$
\begin{aligned}
\frac{d}{d t} \int \psi(\kappa) d \tilde{s} & =\int \psi^{\prime}\left(v_{\tilde{s} \tilde{s}}+\kappa^{2} v\right)-v \kappa \psi(\kappa) d \tilde{s} \\
& =\int\left[-\psi^{\prime \prime}(\kappa) \kappa_{\tilde{s}} v_{\tilde{s}}+\kappa\left(\kappa \psi^{\prime}(\kappa)-\psi(\kappa)\right) v\right] d \tilde{s}
\end{aligned}
$$

Note that $-\psi^{\prime \prime}(\kappa) \leq 0$. If we take for given $\delta>0$,

$$
\psi(\kappa):=\sqrt{\delta+\kappa^{2}},
$$

then $\kappa \psi^{\prime}(\kappa)-\psi(\kappa) \leq 0$, so

$$
\frac{d}{d t} \int \sqrt{\delta+\kappa^{2}} d \tilde{s} \leq 0
$$

Letting $\delta \downarrow 0$, we get that

$$
\frac{d}{d t} \int|\kappa| d \tilde{s} \leq 0
$$

as claimed.

As in $[4,5]$, one can now show that for any classical solution $\left\{\mathcal{C}_{t}: 0<t<T\right\}$, a limit curve $\mathcal{C}_{T}$ exists, and that $\mathcal{C}_{T}$ has finitely many singular points $P_{1}, \ldots, P_{N}$ at which some of the total curvature $\int|\kappa| d \tilde{s}$ must concentrate. Starting at every singular point $P_{i}$ one has two branches of the curve, which we shall refer to as the in- and out-going branch. Since these are limits for $t \uparrow T$ of disjoint $\operatorname{arcs}$ in $\mathcal{C}_{t}$ the strong maximum principle implies that the in- and out-going branches are disjoint unless they coincide, in which case they are part of a line segment ending at $P_{i}$. We refer to this latter situation by saying that $\mathcal{C}_{T}$ has a needle at $P_{i}$.

The following key result holds:

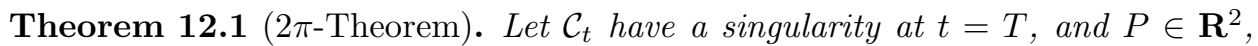
and suppose the in- and out-going branches of $\mathcal{C}_{T}$ at $P$ do not coincide. Then for any $\varepsilon>0$ there is a $t_{\varepsilon}<T$ such that each $\mathcal{C}_{t} \cap B_{\varepsilon}(P)$ with $t_{\varepsilon}<t<T$ has a connected subarc $\beta \subset\left(\mathcal{C}_{t} \cap B_{\varepsilon}(P)\right)$ on which

$$
\left|\int_{\beta} \kappa d \tilde{s}\right| \geq 2 \pi-\varepsilon
$$

Proof. The proof is the same as in [5], Theorem 7.1, with only one modification. Since the strong form of the Sturmian Theorem is not available, we must be careful when we use the foliated rectangle $\mathcal{F}$. But Theorem 11.2 solves this problem. More precisely, assume to the contrary that

$$
\left|\int_{\beta} \kappa d \tilde{s}\right|<2 \pi-\varepsilon
$$

for all subarcs $\beta \subset\left(\mathcal{C}_{t} \cap B_{\varepsilon}(P)\right)$; we construct a foliated rectangle exactly as in [5] (proof of Theorem 7.1). We can then verify that $\mathcal{C}_{t}$ avoids the edges of $\mathcal{F}_{0}$ and passes transversely through $\mathcal{F}_{0}$ once. By Theorem $11.2, \mathcal{C}_{t}$ cannot form a singularity in $\mathcal{F}_{T}$. But $P \in \mathcal{F}_{T}$, a contradiction which proves the theorem.

Thus the arguments of [5] carry through without much modification provided the limiting curve $\mathcal{C}_{T}$ has no needles. The following theorem guarantees that this is always the case. 
Theorem 12.2 (No-Needles Theorem). Notation as in Theorem 12.1. The inand out-going branches at any singular point $P_{i}$ are disjoint.

We present the proof in the next section.

\section{Proof of the NO-NEEdLeS TheOREM}

Points on $\mathcal{C}_{t}$ with tangent parallel to the $x$-axis will be called horizontal points. A horizontal spot is either a horizontal point or else a maximal interval on which the tangent is horizontal (equivalently, a horizontal spot is a connected component of the intersection of $\mathcal{C}_{t}$ with a horizontal line).

Lemma 13.1. For each $t>0$ the number of horizontal spots on $\mathcal{C}_{t}$ is finite. This number is non-increasing.

Proof. Between two consecutive horizontal spots one either has an inflection point, or else the curvature changes by $\pm \pi$. Since the total curvature is finite and since $\mathcal{C}_{t}$ has finitely many inflection points, the number of horizontal spots is bounded.

The number of horizontal spots is the sum over all integers $k$ of the number of sign changes of $\theta(\cdot, t)-k \pi$. For each $k$ this number is non-increasing since $\theta$ satisfies (41). Hence the number of horizontal spots cannot increase.

After a certain time the number of horizontal spots, being a non-negative nonincreasing integer, will remain constant. By Proposition 9.3 the tangent angle at any inflection point is monotonically increasing, so we may assume that for $T-\delta<t<T$ none of the inflection points are horizontal. Thus all horizontal points are located on convex or concave $\operatorname{arcs}$ of $\mathcal{C}_{t}$. Near them the equation (15) is non-degenerate, so $\mathcal{C}_{t}$ is a real analytic curve near each horizontal spot. We can therefore enumerate and trace the horizontal spots $Q_{1}(t), \ldots, Q_{N}(t)$ throughout the time interval $(T-\delta, T)$.

Lemma 13.2. The limits $\lim _{t \uparrow T} Q_{j}(t)=Q_{j}(T)$ exist.

Proof. Let $Q_{j}(t)=\left(x_{j}(t), y_{j}(t)\right)$. Since $Q_{j}(t)$ is horizontal we have just argued that $Q_{j}(t)$ is never an inflection point, and hence that the curvature $\kappa\left(Q_{j}(t), t\right)$ has constant sign. Hence $y_{j}^{\prime}(t)=\sqrt[3]{\kappa\left(Q_{j}(t), t\right)}$ has constant sign and $y_{j}(t)$ converges as $t \uparrow T$.

To prove convergence of $x_{j}(t)$ we use the Chen-Matano [11] device of comparing $\mathcal{C}_{t}$ with its reflection in a vertical line. Suppose

$$
\liminf _{t \uparrow T} x_{j}(t)=\alpha<\beta=\limsup _{t \uparrow T} x_{j}(t) .
$$

Choose $\gamma \in(\alpha, \beta)$ and let $\tilde{\mathcal{C}_{t}}$ be the reflection of $\mathcal{C}_{t}$ in the vertical line $x=\gamma$. Both $\mathcal{C}_{t}$ and $\tilde{\mathcal{C}}_{t}$ are solutions of Affine Curve Shortening, so the number of intersections $\#\left(\mathcal{C}_{t} \cap \tilde{\mathcal{C}_{t}}\right)$ cannot increase. As $t \uparrow T x_{j}(t)=\gamma$ must occur infinitely often. Each time this happens $\mathcal{C}_{t}$ and $\tilde{\mathcal{C}}_{t}$ are tangent at $Q_{j}(t)$, and the number of intersections $\#\left(\mathcal{C}_{t} \cap \tilde{\mathcal{C}}_{t}\right)$ drops. If one chooses $\gamma$ appropriately, $\#\left(\mathcal{C}_{t} \cap \tilde{\mathcal{C}}_{t}\right)$ will be finite for some $t \in(T-\delta, T)\left(\mathcal{C}_{t}\right.$ only has a finite number of vertical spots), so we have a contradiction.

We now turn to the "no-needles theorem". Assume that the in- and out-going branches of a singular point $P_{j} \in \mathcal{C}_{T}$ coincide. Without loss of generality we may assume that $P_{j}$ is the origin, and that the in- and out-going branches both contain an interval $\left[0, \varepsilon_{*}\right]$ on the $x$-axis near $P_{j}$. See Figure 1 . 


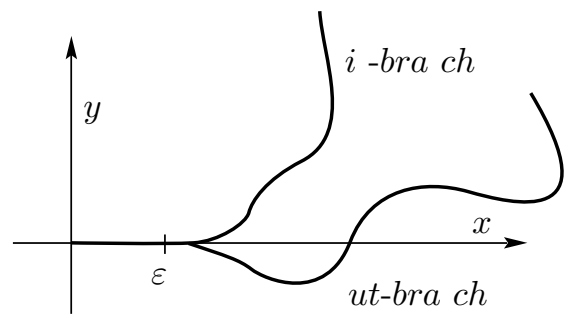

Figure 1. A singular curve with a needle.

In view of Lemma 13.2, we can find $\delta>0$ for any $0<\varepsilon_{1}<\varepsilon_{0}$ such that the strip $\mathcal{S} \stackrel{\text { def }}{=}\left\{(x, y) \mid \varepsilon_{1} \leq x \leq \varepsilon_{2}\right\}$ contains no horizontal points or vertical points for $T-\delta<t<T$. Hence $\mathcal{C}_{t} \cap \mathcal{S}$ consists of a finite number of graphs $y=u^{(k)}(x, t)$, each of which satisfy $u_{t}=\left(u_{x x}\right)^{1 / 3}$. By assumption the $u^{(k)}(x, t)$ tend to zero as $t \uparrow T$.

Lemma 13.3. For each $k$ and all $t \in(T-\delta, T)$, one has

$$
(T-t)^{-3 / 2}\left|u^{(k)}(x, t)\right| \leq \frac{C}{\left(\varepsilon_{0}-x\right)\left(x-\varepsilon_{1}\right)}, \quad \varepsilon_{1}<x<\varepsilon_{0} .
$$

Proof. On the interval $\left(\varepsilon_{1}, \varepsilon_{2}\right)$ the function $u^{(k)}(\cdot, t)$ is monotone - let's assume $u_{x}^{(k)}>0$. If $u^{(k)}\left(x_{0}, t_{0}\right)>0$, then $u^{(k)}\left(x, t_{0}\right) \geq u^{(k)}\left(x_{0}, t_{0}\right)$ for $x \in\left(x_{0}, \varepsilon_{2}\right)$ and hence the graph of $u^{(k)}$ is disjoint from the ellipse inscribed in the rectangle $\left[x_{0}, \varepsilon_{2}\right] \times$ $\left[0, u^{(k)}\left(x_{0}, t_{0}\right)\right]$. Allowing both the curve $\mathcal{C}_{t}$ and the ellipse to evolve by Affine Curve Shortening (15), they must remain disjoint. Since $u^{(k)}(x, t) \rightarrow 0$ as $t \uparrow T$ the ellipse must vanish before $t=T$. This implies that its area is less than $C\left(T-t_{0}\right)^{3 / 2}$ which implies that $u^{(k)}\left(x_{0}, t_{0}\right) \leq C\left(T-t_{0}\right)^{3 / 2} /\left(\varepsilon_{2}-x_{0}\right)$.

If we had started with the assumption that $u^{(k)}(\cdot, t)$ were decreasing rather than increasing we would have arrived at $u^{(k)}\left(x_{0}, t_{0}\right) \leq C\left(T-t_{0}\right)^{3 / 2} /\left(x_{0}-\varepsilon_{1}\right)$. The estimate (55) allows for both possibilities.

Lemma 13.4. For each $k$ the limit

$$
\lim _{t \uparrow T} u^{(k)}(x, t)=\Phi^{(k)}(x)
$$

exists. Moreover, $\Phi^{(k)}(x)$ is a solution of $(50)$.

Proof. Consider

$$
v(x, \tau) \stackrel{\text { def }}{=}(T-t)^{-3 / 2} u^{(k)}(x, t), \quad t=T-e^{-\tau} .
$$

Affine Curve Shortening for the graph of $u^{(k)}$ is equivalent to the following PDE for $v(x, \tau)$ :

$$
\frac{\partial v}{\partial \tau}=\left(v_{x x}\right)^{1 / 3}+\frac{3}{2} v .
$$

Moreover we have just shown in (55) that

$$
|v(x, \tau)| \leq \frac{C}{\left(\varepsilon_{0}-x\right)\left(x-\varepsilon_{1}\right)}, \quad \text { when } \varepsilon_{1}<x<\varepsilon_{0}, \tau>\log \frac{1}{\delta} .
$$


Regularity for (57) implies that $v_{x}, v_{x x}$ and $\left(\left(v_{x x}\right)^{1 / 3}\right)_{x}$ are uniformly bounded for $\tau \uparrow \infty$ on any compact interval $\left[\varepsilon_{1}^{\prime}, \varepsilon_{0}^{\prime}\right] \subset\left(\varepsilon_{1}, \varepsilon_{0}\right)$. (To avoid proving regularity for (57) observe that for any solution $v$ of $(57)$

$$
u(x, t)=(1-t)^{3 / 2} v\left(x, \tau_{0}-\log [2(1-t)]\right)
$$

satisfies $u_{t}=\left(u_{x x}\right)^{1 / 3}$; use the results of Section 8 to obtain regularity for $u$ for $0<t<3 / 4$ and then transfer to $v$ to obtain regularity for $\tau_{0}-\log 2<\tau<\tau_{0}+\log 2$.)

These estimates imply that any sequence $\tau_{j} \uparrow \infty$ has a subsequence along which $v\left(x, \tau_{j_{k}}+\tau\right)$ converges to some eternal solution $v^{*}(x, \tau)$ (i.e., defined for $\varepsilon_{1}<x<\varepsilon_{0}$ and all $\tau \in \mathbf{R}$ ) of (57). To complete our proof we will now show by "intersection comparison" that $v^{*}$ is in fact time independent, and hence a solution of (50) as claimed.

Consider the curves

$$
\hat{\mathcal{C}}_{\tau}=\left\{\left(x,(T-t)^{-3 / 2} y\right) \mid(x, y) \in \mathcal{C}_{t}\right\},
$$

where $T-t=e^{-\tau}$. The graph of $v\left(\cdot, \tau_{j_{k}}\right)$ is a part of $\hat{\mathcal{C}}_{\tau_{j_{K}}}$.

Proposition 13.5. Let $\tau_{0} \in \mathbf{R}$. Let $\Phi_{*}$ be any solution of (50). Then one can approximate $\Phi_{*}$ by other solutions $\Phi_{i} \rightarrow \Phi_{*}$ of (50) which intersect $\hat{\mathcal{C}}_{\tau_{0}}$ only finitely often.

Since the $\Phi_{i}$ are steady states for (57) the number of intersections of $\Phi_{i}$ with $\hat{\mathcal{C}}_{\tau}$ does not increase with $\tau$, and it follows in particular that the limit $v^{*}(\cdot, \tau)$ only intersects $\Phi_{i}$ finitely often.

Proof. For solutions $\Phi$ of (50) the quantity $E_{\Phi}:=\left(\Phi^{\prime}\right)^{2}-\frac{27}{16} \Phi^{4}$ is constant.

Let $\mathcal{C}^{\prime}$ denote $\hat{\mathcal{C}}_{\tau_{0}}$ with the vertical points deleted. The curve $\mathcal{C}^{\prime}$ is a finite union of graphs $y=\bar{v}(x)$. We consider the function $E=\left(\bar{v}_{x}\right)^{2}-\frac{27}{16} \bar{v}^{4}$. Since $\bar{v}_{x} \rightarrow \pm \infty$ at the vertical points, $E: \mathcal{C}^{\prime} \rightarrow \mathbf{R}$ is a $C^{1}$ and proper function. By Sard's theorem the regular values of $E$ form an open and dense subset of the real line.

If the "energy" $E_{\Phi_{*}}$ of the given solution $\Phi_{*}$ is not a regular value, then one can approximate $E_{*}$ with regular values $E_{i} \rightarrow E_{*}$, and select corresponding solutions $\Phi_{i} \rightarrow \Phi_{*}$ of (50).

At a tangency of $\Phi_{i}$ and $\mathcal{C}^{\prime}$ one has $E=E_{i}$. Since $E_{i}$ is a regular value of $E: \mathcal{C}^{\prime} \rightarrow \mathbf{R}$, there are only finitely many such tangencies. All other intersections of $\Phi_{i}$ and $\mathcal{C}^{\prime}$ must be transverse. Hence there only finitely many such intersections.

We return to the proof of Lemma 13.4. Assume that for some $\left(x_{0}, \tau_{0}\right)$ one has

$$
v^{*}\left(x_{0}, \tau_{0}\right) \neq 0 \text { and } v_{\tau}^{*}\left(x_{0}, \tau_{0}\right) \neq 0 .
$$

Let $\Phi$ be the solution of (50) with $\Phi\left(x_{0}\right)=v^{*}\left(x_{0}, \tau_{0}\right)$ and $\Phi^{\prime}\left(x_{0}\right)=v_{x}^{*}\left(x_{0}, \tau_{0}\right)$. By (58) we then have $\Phi^{\prime \prime}\left(x_{0}\right) \neq 0$ and $\Phi^{\prime \prime}\left(x_{0}\right) \neq v_{x x}^{*}\left(x_{0}, \tau_{0}\right)$. It follows that $\Phi(\cdot)-$ $v^{*}(\cdot, \tau)$ loses exactly two zeroes at time $\tau=\tau_{0}$. In fact for $\tilde{\Phi}$ close to $\Phi$ and for any $\tilde{v}$ with $\tilde{v}, \tilde{v}_{\tau}$, and $\tilde{v}_{x x}$ uniformly close to $v^{*}, v_{\tau}^{*}$, and $v_{x x}^{*}$, respectively, the difference $\tilde{v}-\tilde{\Phi}$ will also lose at least two zeroes near $\left(x_{0}, \tau_{0}\right)$. In particular we could take $\tilde{v}(x, \tau)=v^{*}\left(x, \tau+\tau_{j_{k}}\right)$ with $k$ large and we could choose a $\tilde{\Phi}$ near $\Phi$ for which $\hat{\mathcal{C}}_{\tau}$ only intersects $\tilde{\Phi}$ finitely often. Then the number of zeroes of $v^{*}(\cdot, \tau)-\Phi(\cdot)$ would drop infinitely often, which is impossible.

Thus the assumption (58) is incorrect, and we find that $v^{*}(x, \tau) \equiv \Phi(x)$ for some solution $\Phi$ of (50). 


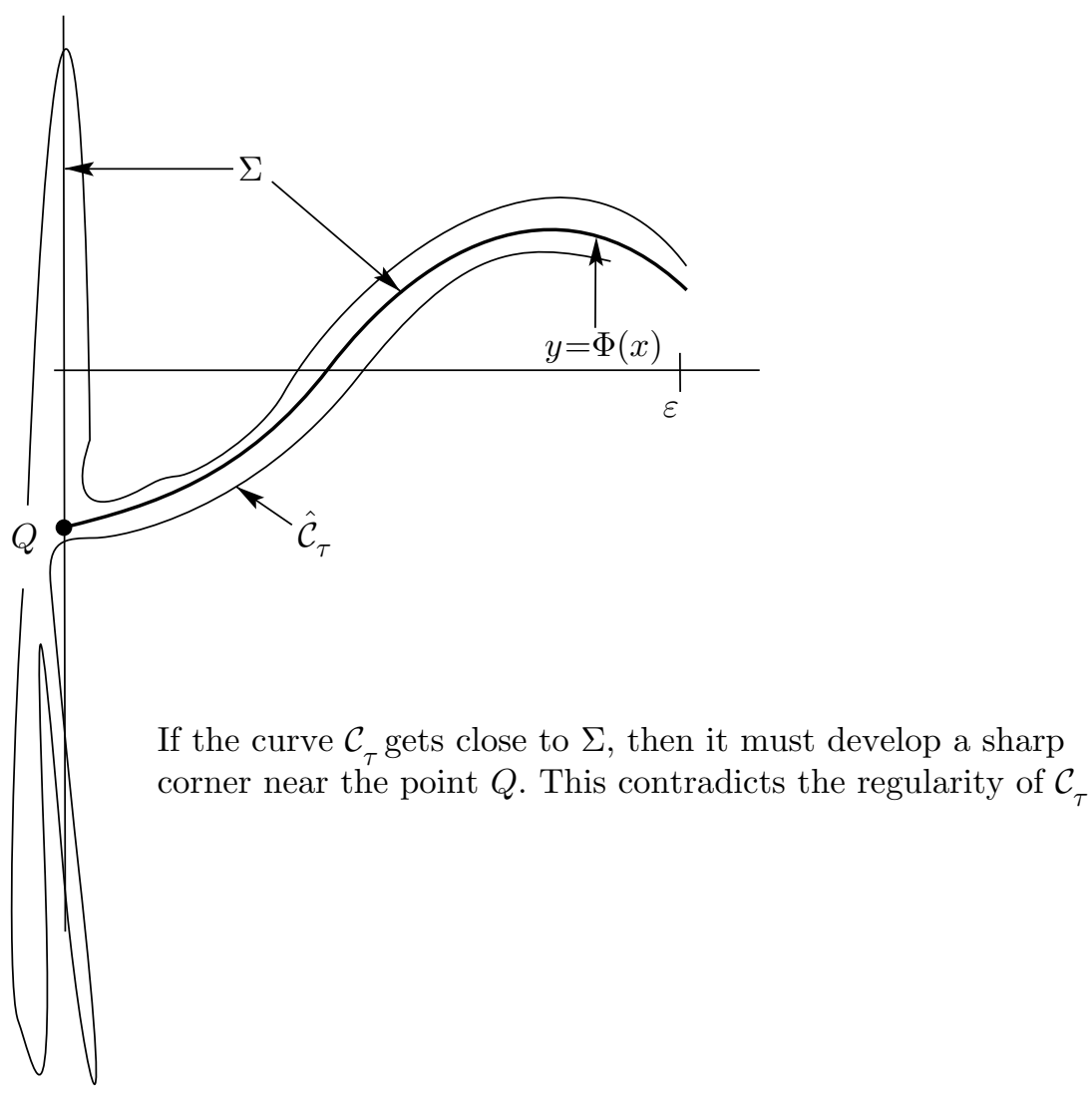

Figure 2. $\Sigma$ and the curve $\hat{\mathcal{C}}_{\tau}$ converging to $\Sigma$.

We now conclude the proof of the "no-needles theorem." Let $\tau_{j} \uparrow \infty$ be such that $v\left(x, \tau+\tau_{j}\right)$ converges uniformly for bounded $\tau$ and on compact intervals $\left[\varepsilon_{1}, \varepsilon_{0}\right] \subset$ $\left(0, \varepsilon_{*}\right)$ to some solution $\Phi$ of $(50)$.

Let $\Sigma$ be the union of the $y$-axis and the curve $\{(x, \Phi(x)) \mid x \geq 0\}$.

As $j \rightarrow \infty$, the part of the curves $\hat{\mathcal{C}}_{\tau}$ in the strip $\left\{(x, y) \mid-\varepsilon_{*}<x<\varepsilon_{*}\right\}$ converge to $\Sigma$. This forces $\hat{\mathcal{C}}_{\tau}$ to have a sharp corner near the point where the graph of $\Phi$ intersects the $y$-axis. See Figure 2. But $\hat{\mathcal{C}}$ evolves by

$$
v=\kappa^{1 / 3}+\frac{3}{2} y \cos \theta
$$

and one easily deduces regularity results for this equation from similar results for $v=\kappa^{1 / 3}$. In particular, $\hat{\mathcal{C}}_{\tau}$ must have uniformly bounded curvature in bounded regions of the $x y$-plane. It is therefore impossible for $\hat{\mathcal{C}}_{\tau}$ to converge to $\Sigma$.

\section{The AFFine $\delta$-WHiSKer LEMMA}

In this section, we recall Grayson's $\delta$-whisker lemma [16] and point out that it is also valid for the affine shortening flow (15). The $\delta$-whisker lemma prevents a curve from getting too close to itself along subarcs which turn through at least $\pi$. 
Since the proof in this affine case is very similar to that of [16], we will only sketch the relevant details.

14.1. $\alpha$-points. Let $\left\{\mathcal{C}_{t} \mid 0<t<T\right\}$ be a maximal solution of Affine Curve Shortening (15), and choose $T_{0}<T$ so that the (finite) number of convex and concave arcs remains constant for $T_{0} \leq t<T$.

For $\alpha \in \mathbf{R}$ we define an $\alpha$-point on $\mathcal{C}_{t}$ to be a point where $\theta(p, t)=\alpha(\bmod 2 \pi)$. An $\alpha$-spot is, by definition, a connected component of the set of $\alpha$-points. By applying Lemma 13.1 in a suitably rotated coordinate system we conclude that at any given instant $t \in(0, T)$ there exists at most a finite number of $\alpha$-spots, and that this number is non-increasing in time.

Proposition 14.1. Let $T_{0}<t_{1}<T$. If $A_{1} \in \mathcal{C}_{t_{1}}$ is an $\alpha$-point, but not an inflection point, then there exists a smooth family $\left\{A_{t} \mid T_{0} \leq t \leq t_{1}\right\}$ of $\alpha$-points with $A_{t_{1}}=A_{1}$.

Proof. Since $A_{1}$ is not an inflection point, the evolution is smooth near $\left(A_{1}, t_{1}\right)$ in space-time. At a non-inflection point one has $\theta_{\tilde{s}}=\kappa \neq 0$ so the Implicit Function Theorem provides a smooth family of points $A_{t}$ with $\theta\left(A_{t}, t\right)=\alpha$. This family may only be defined for $t$ close to $t_{1}$ : let $\left(t_{2}, t_{1}\right]$ be the largest interval on which such a smooth family can be defined. Then $A_{t}$ will accumulate at inflection spots of $\mathcal{C}_{t_{2}}$ as one lets $t$ decrease to $t_{2}$.

By Proposition 9.3 the only way an inflection spot can become an $\alpha$-spot is for two $\alpha$-points with non-zero curvature to meet and annihilate each other. Thus a small neighborhood of an inflection spot at time $t_{2}$ which is also an $\alpha$-spot will not contain any $\alpha$-points for $t$ slightly above $t_{2}$. Consequently, if one traces a smooth family of $\alpha$-points $A_{t}$ back in time it can never run into an inflection spot with $t_{2} \geq T_{0}$. Hence we can continue our smooth family of $\alpha$-points all the way back to $T_{0}$.

14.2. $\alpha$-arcs. Following Grayson [16, page 295] we define an $\operatorname{arc} \mathcal{B} \subset \mathcal{C}_{t_{0}}$ to be an $\alpha$-arc ${ }^{1}$ if the inward pointing unit tangent vectors to the arc are both given by $\left(\begin{array}{c}\cos \alpha \\ \sin \alpha\end{array}\right)$.

Thus the endpoints of an $\alpha$-arc are $\alpha$ - or $\alpha+\pi$-points. As we have seen, these can be traced back in time as long as $t \geq T_{0}$. The resulting family of $\alpha$-arcs $\left\{\mathcal{B}_{t} \mid T_{0} \leq t \leq t_{0}\right\}$ will be called the history of $\mathcal{B}_{t_{0}}$.

Theorem 14.2 ( $\delta$-Whiskers). There exists a $\delta>0$ such that for any point $P$ on an $\alpha$-arc $\mathcal{B}_{t_{0}} \subset \mathcal{C}_{t_{0}}$ with $t_{0} \in\left[T_{0}, T\right)$, the line segment

$$
\ell_{P, \delta, \alpha} \stackrel{\text { def }}{=}\left\{P+r \mathbf{v}_{\alpha} \mid 0 \leq r \leq \delta\right\}, \quad \mathbf{v}_{\alpha}=\left(\begin{array}{c}
\cos \alpha \\
\sin \alpha
\end{array}\right),
$$

is disjoint from $\mathcal{C}_{t_{0}} \backslash \mathcal{B}_{t_{0}}$.

To prove this one traces the history of $\mathcal{B}_{t_{0}}$ back to time $t=T_{0}$. For $d>0$ and $T_{0} \leq t \leq t_{0}$ we then define $\mathcal{B}_{t}^{d}$ to be $\mathcal{B}_{t}$ translated by $d \cdot \mathbf{v}_{\alpha}$. We also define $d(t)$ to be the largest $d>0$ such that $\mathcal{B}_{t}^{d^{\prime}}$ is disjoint from $\mathcal{C}_{t} \backslash \mathcal{B}_{t}$ for all $0<d^{\prime}<d$.

At $t=T_{0}$ a compactness argument provides a $\delta$ independent of the arc such that one can translate $\mathcal{B}_{T_{0}}$ by an amount $\delta$ in the direction of the vector $\mathbf{v}_{\alpha}$ without bumping into $\mathcal{C}_{T_{0}} \backslash \mathcal{B}_{T_{0}}$, i.e., such that $d\left(T_{0}\right) \geq \delta$.

\footnotetext{
${ }^{1}$ Grayson calls these arcs "nice".
} 
Grayson then shows, using maximum principle arguments, that $d(t)$ is a nonincreasing function. The $\operatorname{arcs} \mathcal{B}_{t}^{d(t)}$ and $\mathcal{C}_{t} \backslash \mathcal{B}_{t}$ must have a tangency. If the tangency is interior, then it will disappear instantaneously by the strong maximum principle (see Lemma 7.1). If the tangency occurs at an endpoint of $\mathcal{B}_{t}^{d(t)}$, then the curvatures of $\mathcal{B}_{t}^{d(t)}$ and $\mathcal{C}_{t} \backslash \mathcal{B}_{t}$ must be different (for otherwise $\mathcal{C}_{t} \backslash \mathcal{B}_{t}$ would intersect the $d(t)$ whisker $\ell_{P, d(t), \alpha}$ where $P$ is the corresponding endpoint on $\mathcal{B}_{t}$ ). Hence the tangency disappears in this case as well, and $d(t)$ is indeed non-decreasing.

\section{Convergence to a point}

Our main result is:

Theorem 15.1. Let $\left\{\mathcal{C}_{t} \mid 0 \leq t<T\right\}$ be a maximal classical solution of Affine Curve Shortening (15). Then

(1) $\mathcal{C}_{t}$ shrinks to a point as $t \uparrow T$, and

(2) The total curvature $\int|\kappa| d \tilde{s}$ of $\mathcal{C}_{t}$ tends to $2 \pi$ as $t \uparrow T$.

To prove this we argue by contradiction: assume the limit curve $\mathcal{C}_{T}$ is not a single point, and let $P$ be one of the singular points on this curve. By the $2 \pi$-Theorem 12.1 there exist $t_{n} \uparrow T$ such that $\mathcal{C}_{t_{n}} \cap B\left(P, 2^{-n}\right)$ contains an arc $\mathcal{B}_{n}$ with turning angle

$$
\left|\int_{\mathcal{B}_{n}} \kappa d \tilde{s}\right| \geq \frac{3}{2} \pi
$$

This puts us in Grayson's "case I" (see [16, page 300]). In [16, Theorem 4.1] he shows that this case cannot occur, and the two ingredients of his proof are " $\delta$ whiskers" and our Lemma 9.10 which states that arcs with turning angle less than $\pi$ have uniformly bounded curvature. As we have verified these statements for Affine Curve Shortening, Grayson's arguments lead us to a contradiction. The curve therefore must shrink to a point.

The second statement of the theorem is Grayson's Lemma 3.9, whose proof also is based on Lemma 9.10 and $\delta$-whiskers. The same arguments therefore apply in our setting.

\section{REFERENCES}

[1] L. Alvarez, F. Guichard, P. L. Lions, and J. M. Morel, "Axiomes et equations fondamentales du traitement d'images," C. R. Acad. Sci. Paris 315, pp. 135-138, 1992. MR 94d:47066

[2] L. Alvarez, F. Guichard, P. L. Lions, and J. M. Morel, "Axiomatisation et nouveaux operateurs de la morphologie mathematique," C. R. Acad. Sci. Paris 315, pp. 265-268, 1992. MR 94d:47067

[3] B. Andrews, "Contraction of convex hypersurfaces by their affine normal," J. Differential Geometry 43, pp. 207-230, 1996. MR 97m:58045

[4] S. Angenent, "Parabolic equations for curves on surfaces, Part I. Curves with $p$-integrable curvature," Annals of Mathematics 132, pp. 451-483, 1990. MR 91k:35102

[5] S. Angenent, "Parabolic equations for curves on surfaces, Part II. Intersections, blow-up, and generalized solutions," Annals of Mathematics 133, pp. 171-215, 1991. MR 92b:58039

[6] S. Angenent, "On the formation of singularities in the curve shortening flow," J. Differential Geometry 33, pp. 601-633, 1991. MR 92c:58016 
[7] S. Angenent, "The zero set of a solution of a parabolic equation," J. für die reine and angewandte Mathematik 390, pp. 79-96, 1988. MR 89j:35015

[8] W. Blaschke, Vorlesungen über Differentialgeometrie II, Verlag Von Julius Springer, Berlin, 1923.

[9] S. Buchin, Affine Differential Geometry, Gordon and Breach, Science Publishers, Inc., New York, 1983. MR 85g:53010

[10] J. Dieudonné and J. Carrell, Invariant Theory: Old and New, Academic Press, London, 1970. MR 43:4828

[11] X.-Y. Chen and H. Matano, "Convergence, asymptotic periodicity and one point blow-up in one dimensional semilinear heat equations," Journal of Differential Equations 33, pp. 160-190 (1989). MR 90e:35018

[12] C. L. Epstein and M. Gage, "The curve shortening flow," in Wave Motion: Theory, Modeling, and Computation, A. Chorin and A. Majda, Editors, Springer-Verlag, New York, 1987. MR 89f: 58128

[13] M. Gage, "An isoperimetric inequality with applications to curve shortening," Duke Mathematical Journal 50, pp. 1225-1229, 1983. MR 85d:52007

[14] M. Gage, "Curve shortening makes convex curves circular," Invent. Math. 76, pp. 357-364, 1984. MR 85i:52004

[15] M. Gage and R. S. Hamilton, "The heat equation shrinking convex plane curves," J. Differential Geometry 23, pp. 69-96, 1986. MR 87m:53003

[16] M. Grayson, "The heat equation shrinks embedded plane curves to round points," J. Differential Geometry 26, pp. 285-314, 1987. MR 89b:53005

[17] M. Grayson, "Shortening embedded curves," Annals of Mathematics 129, pp. 71-111, 1989. MR 90a:53050

[18] H. W. Guggenheimer, Differential Geometry, McGraw-Hill Book Company, New York, 1963. MR 27:6194; MR 58:12737

[19] B. B. Kimia, A. Tannenbaum, and S. W. Zucker, "On the evolution of curves via a function of curvature, I: the classical case," J. of Math. Analysis and Applications 163, pp. 438-458, 1992. MR 93a:58037

[20] B. B. Kimia, A. Tannenbaum, and S. W. Zucker, "Shapes, shocks, and deformations," Int. J. Computer Vision 15 (1995), 189-224.

[21] H. Matano, "Non-increase of the lapnumber of a solution for a one dimensional semilinear parabolic equation," J. Fac. Sci. Univ. Tokyo Math. 29 (1982), 401-441. MR 84m:35060

[22] P. J. Olver, Applications of Lie Groups to Differential Equations, Second Edition, SpringerVerlag, New York, 1993. MR 94g:58260

[23] P. J. Olver, "Differential invariants," to appear in Acta Appl. Math.

[24] P. Olver, G. Sapiro, and A. Tannenbaum, "Differential invariant signatures and flows in computer vision: A symmetry group approach," Geometric Driven Diffusion, edited by Bart ter har Romeny, Kluwer, 1994.

[25] P. Olver, G. Sapiro, and A. Tannenbaum, "Classification and uniqueness of invariant geometric flows," Comptes Rendus Acad. Sci. (Paris) 319 (1994), 339-344. MR 95j:58180

[26] P. Olver, G. Sapiro, and A. Tannenbaum, "Invariant geometric evolutions of surfaces and volumetric smoothing," SIAM J. Applied Math. 57 (1997), 176-194. MR 97j:53014

[27] P. Olver, G. Sapiro, and A. Tannenbaum, "Affine invariant edge maps and active contours," to appear in $C V I U$.

[28] S. J. Osher and J. A. Sethian, "Fronts propagation with curvature dependent speed: Algorithms based on Hamilton-Jacobi formulations," Journal of Computational Physics 79, pp. 12-49, 1988. MR 89h:80012

[29] M. Protter and H. Weinberger, Maximum Principles in Differential Equations, Prentice-Hall, New York, 1967. MR 36:2935

[30] G. Sapiro and A. Tannenbaum, "On affine plane curve evolution," Journal of Functional Analysis 119, pp. 79-120, 1994. MR 94m:58049

[31] G. Sapiro and A. Tannenbaum, "Affine invariant scale-space," Int. J. Computer Vision 11, pp. 25-44, 1993.

[32] G. Sapiro and A. Tannenbaum, "Invariant curve evolution and image processing," Indiana Univ. Journal of Math. 42, pp. 985-1009, 1993. MR 94m:58048

[33] G. Sapiro and A. Tannenbaum, "Area and length preserving geometric invariant scale-spaces," IEEE Trans. Pattern Analysis and Machine Intelligence 17 (1995), 1066-1070. 
[34] M. Spivak, A Comprehensive Introduction to Differential Geometry, Publish or Perish Inc, Berkeley, California, 1979. MR 82g:53003a; MR 82g:53003b; MR 82g:53003c; MR 82g:53003d; MR 82g:53003e

[35] B. White, "Some recent developments in differential geometry," Mathematical Intelligencer 11, pp. 41-47, 1989. MR 90k:53003

(S. Angenent) Department of Mathematics, University of Wisconsin, Madison, WisCONSIN 53706

(G. Sapiro and A. Tannenbaum) Department of Electrical and Computer Engineering, University of Minnesota, Minneapolis, Minnesota 55455

E-mail address: tannenba@ece.umn.edu 